\title{
La qualité étatique accordée par le juge interne : Une reconnaissance procédurale de l'État?
}

\section{Mehdi Belkabla"}

«La reconnaissance en ce qui concerne les facultés juridiques que le droit commun international attribue [...] n'a pas besoin d'intervenir lorsque ces facultés ne sont pas contestées. En ce cas, elle sera l'œuvre d'un tribunal $[\ldots] »^{1}$

\section{Introduction}

\section{A. La reconnaissance : des enjeux multiples}

La question de la reconnaissance, institution classique du droit international, ${ }^{2}$ constitue l'un des sujets les plus fascinants de la discipline. ${ }^{3}$ Cette fascination a pu toutefois être supplantée par une forme de frustration, comme en témoignent les propos de J. Dugard selon lequel « [t $t$ out juriste prétendant examiner les mystères de la [...] reconnaissance et ayant pour dessein $\mathrm{d}[$ 'en] fournir une explication cohérente [...] dans le cadre d'une théorie juridique s'exposera immanquablement à la dérision et à la vitupération $» .{ }^{4}$ L'expression de son scepticisme n'a évidemment pas entamé sa témérité ni celle de nombreux membres de la doctrine, puisque la reconnais-

* Research Fellow à l'Institut Max Planck Luxembourg pour le droit procédural ; Doctorant à l'Université Panthéon-Assas (Paris II).

1 G. Scelle, Règles générales du droit de la paix, 46 R.C.A.D.I. (1933), 327, 379.

$2 \mathrm{~J}$. Verhoeven, Les relations internationales de droit privé en l'absence de reconnaissance d'un État, d'un gouvernement ou d'une situation, 192 R.C.A.D.I. (1985), 9, 19 [Verhoeven, Les relations internationales] ; R. Y. Jennings, General Course on Principles of International Law, 121 R.C.A.D.I. (1967), 323, 349 ; J. Chatelain, La reconnaissance internationale, in $\mathrm{Ch}$. Rousseau (dir.), La technique et les principes en droit public : Études en l'honneur de Georges Scelle (1950), 717, 719 : « [...] [C]ertains [...] [ont] vu [dans la notion de reconnaissance] "l'institution fondamentale du Droit des gens" ».

$3 \mathrm{~J}$. Verhoeven, La reconnaissance internationale dans la pratique contemporaine : les relations publiques internationales (1975), 1 [Verhoeven, Reconnaissance].

4 J. Dugard, Recognition and the United Nations (1987), 5 [notre traduction]. 
sance fait encore et toujours l'objet d'une attention particulière. Il apparaît, à cet égard, que trois qualités propres à la question fondent l'intérêt que continuent de lui porter les internationalistes : sa juridicité, sa complexité et son actualité.

Le débat sur l'appartenance de l'institution de la reconnaissance à la discipline juridique n’a jamais cessé d'agiter la doctrine. Certains réalistes affirment ainsi sans ambages son ancrage dans la sphère des relations internationales et de la science politique. ${ }^{5}$ Et, il faut en convenir, il serait illusoire de tenter d'imposer un apolitisme absolu à la reconnaissance sans s'écarter dangereusement d'une vérité éprouvée et approuvée. ${ }^{6}$ Néanmoins, si d'aucuns ont pu insister plus que de raison sur la prépondérance du politique sur l'ensemble des étapes menant à l'adoption de l'acte de reconnaissance et à ses conséquences, ${ }^{7}$ dans leur immense majorité, les internationalistes ont su justifier l'intérêt porté à la reconnaissance par la science juridique. ${ }^{8}$ La position de I. Brownlie est à cet égard tout à fait à propos. Il affirme que bon nombre d'aspects attachés à la reconnaissance ont incontestablement une dimension politique, mais pas davantage que toute autre

5 H. Lauterpacht, Recognition of States in International Law, 53 Yale Law Journal (1944), 385, 386 [Lauterpacht, Recognition of States] : « La majorité des auteurs adhèrent à l'idée que l'acte de reconnaissance en tant que tel n'est pas une matière régie par le droit, mais une question de nature politique " [notre traduction] ; R. Bierzaneck, La non-reconnaissance et le droit international contemporain, 8 Annuaire français de droit international (1962), 117, 124 : «On a exprimé bien des fois l'opinion que la reconnaissance, étant un acte de caractère purement politique, ne relève point du droit international ».

6 C. Hillgruber, The Admission of New States to the International Community, 9 European Journal of International Law (1998), 491 : «[...] [L]'importance du caractère politique de la reconnaissance d'État est indéniable [...] » [notre traduction] ; Jennings, supra note 2, 356 : "En tout état de cause, il y a un manque certain de réalisme de la part des doctrines prônant le fait que les décisions [de reconnaissance] puissent être prises en vertu de motifs purement juridiques sans avoir égard à des motivations politiques » [notre traduction].

7 J. Verhoeven, Droit international public (2000), 74 ; H. Fromageot, Comité d'experts pour la codification progressive du droit international de la Société des Nations, Procès-verbaux de la première session, 7 mai 1925, 40 : «La reconnaissance [...] n'est pas une matière qui puisse être réglée juridiquement ; elle est exclusivement d'ordre politique ".

8 E. Wyler, Théorie et pratique de la reconnaissance d'État : une approche épistémologique du droit international (2013), xvi [E. Wyler, Théorie et pratique] : "Nous ne croyons pas que la fécondité d'une analyse politique exclue toute approche juridique $»$. 
question intéressant le droit international. ${ }^{9}$ Il s'ensuit que les scientifiques du droit seraient bien affligés s'ils devaient se détourner d'un objet d'étude uniquement parce que celui-ci présenterait des traits extra-juridiques.

Les discussions entourant la question de la juridicité de la reconnaissance laissent poindre toute la complexité du sujet. Il règne, en effet, une quasi-unanimité au sein de la doctrine sur la reconnaissance des difficultés posées par la reconnaissance. ${ }^{10}$ Les vocables ne manquent pas pour témoigner des obstacles que le juriste doit surmonter pour ne serait-ce que s'essayer à une définition de la notion. Nombreux sont ceux qui parlent de la " confusion " ${ }^{11}$ qui enserre la question, tandis que d'autres évoquent de façon plus emphatique " un fardeau de difficultés et de malentendus ${ }^{12}$ en la matière. La rudesse de l'étude de ce sujet a ainsi pu autoriser de nombreuses tentatives de (re-)théorisation. ${ }^{13}$

Ces ambitions doctrinales sont également le corollaire de l'actualité de la question. Récemment, la reconnaissance, sous sa variante étatique ou gouvernementale, a fait l'objet d'un regain d'intérêt au vu des développements qui secouent la société internationale. La série de changements révolutionnaires de gouvernement dans le monde arabe, les velléités de l'Autorité palestinienne d'intégrer certaines institutions internationales ou en-

9 I. Brownlie, Recognition in Theory and Practice, 53 British Yearbook of International Law (1982), 197, 201 ; voir également, R. Lapidoth et K. N. Calvo-Goller, Les éléments constitutifs de l'État et la déclaration du Conseil national palestinien du 15 novembre 1988, 96 RGDIP (1992), 777, 801 : «La reconnaissance obéit principalement à des mobiles politiques, elle ne constitue pas moins un acte juridique ».

10 S. Talmon, Recognition of Governments in International Law: With Particular Reference to Governments in Exile (1998), 21.

11 Ch. H. Alexandrowicz-Alexander, The Quasi-Judicial Function in Recognition of States and Governments, 46 American Journal of International Law (1952), 631 : «[...] [Une certaine] confusion [...] entour[e] les débats [...] sur la question » [notre traduction]; I. Brownlie, supra note 9, $197:$ : La confusion [...] règne [sur la question] " [notre traduction] ; H. Kelsen, Recognition in International Law: Theoretical Observations, 35 American Journal of International Law (1941), 605, 606 [Kelsen, Recognition] : « [...] [L]a confusion prév[aut]» [notre traduction]; Bierzaneck, supra note $5,123:$ « La doctrine $[\ldots]$ en matière de reconnaissance est confuse et divergente [...]».

12 F. Münch, Quelques problèmes de la reconnaissance en droit international, in Miscellanea Walter Jean Ganshof Van Der Meersch: studia ab discipulis amicisque in honorem egregii professoris edita (1972), 157, 167.

13 En langue française, une des études de référence est celle de J. Charpentier, La reconnaissance internationale et l'évolution du droit des gens (1956). Pour une étude récente, voir $\mathrm{E}$. Wyler, Théorie et pratique, supra note 8. 
core la question controversée des quasi-États ${ }^{14}$ représentés par des entités aussi diverses que Taïwan, le Kosovo ou la Transnistrie sont en effet autant de problématiques qui, sans être réduites à la question de la reconnaissance, ont un rapport évident avec celle-ci.

D’autres enjeux non pas inédits mais remarquables ont pu récemment être relevés dans le cadre de la reconnaissance. En effet, il semblerait que le monopole dont pouvaient jouir les exécutifs internes ${ }^{15}$ en la matière s'est vu bousculé par des initiatives parlementaires ${ }^{16}$ et des incursions judiciaires. ${ }^{17}$ Notre contribution se proposera d'étudier les cas, relativement nombreux, où la question de la qualité étatique d'une entité se pose au cours de l'instance, et plus particulièrement les affaires dans lesquelles le juge interne accepte effectivement de se prononcer sur celle-ci en mettant en œuvre une opération juridique spécifique. Certains commentateurs ont pu qualifier très tôt cette pratique de "reconnaissance judiciaire ». ${ }^{18}$ De la même manière, nous pourrons envisager dans quelle mesure l'on pourra parler ou non, dans ces hypothèses, d'une (non-)reconnaissance procédurale ${ }^{19}$ de l'État. Aussi conviendra-t-il de confronter ce phénomène à l'institution

14 P. Kolstø, The Sustainability and Future of Unrecognized Quasi-States, 43 Journal of Peace Research (2006), 723.

15 J. Charpentier, La reconnaissance internationale et l'évolution du droit des gens, supra note 13, 3 : «Ce sont les États qui reconnaissent, par l'organe de leurs gouvernements et plus spécialement du pouvoir exécutif ».

16 Certains parlements ont récemment adopté des résolutions appelant leur gouvernement à reconnaître un État palestinien ; voir p. ex., Assemblée nationale de la République française, Résolution portant sur la reconnaissance de l'État de Palestine, $\mathrm{n}^{\circ} 439,2$ décembre 2014.

17 Certains auteurs ont pu relever une nette tendance contemporaine de la part de certaines juridictions internes à faire fi de l'absence de reconnaissance par l'exécutif dans la résolution des litiges qui se présentent devant elles. Cette situation semble être le fruit d'une lente évolution ; voir à ce sujet, F. Couveinhes-Matsumoto, L'effectivité en droit international (2014), 274-284, paras 266-273.

18 A. D. McNair, Judicial Recognition of States and Governments and the Immunities of Public Ships, 2 British Yearbook of International Law (1921), 57 ; F. A. Mann, The Judicial Recognition of an Unrecognised State, 36 International and Comparative Law Quarterly (1987), 348 ; Verhoeven, Les relations internationales, supra note 2, $23:$ : [...] [D]'aucuns n'ont point hésité à affirmer l'existence d'une reconnaissance en quelque sorte judiciaire, qui exprimerait $[. .$.$] la reconnaissance$ autonome d'un juge [...]».

19 Expression inspirée de la notion « d'État procédural » forgée par E. Wyler, Le droit de la succession d'États à l'épreuve de la fiction juridique, in G. Distefano et al. (dir.), La Convention de Vienne de 1978 sur la succession d'États en matière de traités - Commentaire article par article et études thématiques (2016), 1607, 1655, para 54, [E. Wyler, La succession d'États]. Cette expression permettra également 
juridique de la reconnaissance telle qu'elle est classiquement admise en droit international comme acte juridique unilatéral prononcé par le gouvernement d'un État ; acte auquel sont attachées des conséquences juridiques déterminées. Si la formulation du problème en ces termes peut, dès l'abord, faire douter de la pertinence de la qualification de reconnaissance pour décrire lesdits phénomènes ${ }^{20}$ - notamment au regard de la théorie des actes unilatéraux et plus encore du monopole de l'exécutif dans la conduite des relations internationales -, l'étude exigera, en tout état de cause et au préalable, une tentative de définition de la reconnaissance.

\section{B. La reconnaissance : éléments de définition}

Il apparaît d'emblée que la reconnaissance est une catégorie générale du droit qui ne saurait se limiter à la seule reconnaissance de gouvernement ou d'État $;^{21}$ déclinaison ayant la préférence des auteurs de traités de droit international. Elle peut, à ce titre, se rapporter à maints objets en droit international public 22 : la reconnaissance d'un traité, ${ }^{23}$ d'une responsabilité internationale, ${ }^{24}$ d'une zone de pêche avec droits préférentiels, ${ }^{25}$ etc. Dans cette perspective, M. Jones définit la reconnaissance, "dans un sens générique et large [comme] toute acceptation [...] d'une situation [...] internationale [...] formulée par l'organe exécutif d'un État [...] de manière à $\left[l^{\prime}\right]$ engager $[\ldots] \gg{ }^{26}$ Dans cette formulation, comme dans d'autres, trois élé-

de ne pas confondre le phénomène étudié avec la notion de reconnaissance de facto. Voir à ce sujet, infra note 55 .

20 Verhoeven, Les relations internationales, supra note 2, 23.

21 Brownlie, supra note 9, 200-202.

22 Jennings, supra note 2, 349 : «[...] [S]es domaine[s] d'application [sont] extrêmement large[s] » [notre traduction] ; J. Combacau et S. Sur, Droit international public, $11^{\grave{e}}$ éd. (2014), 289 : «Elle connaît des applications dans la plupart des domaines du droit international ; tout ou presque peut faire l'objet de reconnaissance $[\ldots]$ " ; J. Charpentier, Reconnaissance ( 1 ère publication 1998, actualisation 2009), Répertoire Dalloz de Droit International, para 2 : «Les objets de la reconnaissance sont [...] très divers »; J. Chatelain, La reconnaissance internationale, supra note 2, 717 : « [La reconnaissance] a [...], par sa nature même, un champ d'application extrêmement vaste et s'étend à toute une série d'hypothèses [...] ».

23 Traité de Versailles, 28 juin 1919, Art. 231, 225 CTS 188.

24 Huilca Tecse c. Pérou, CIADH arrêt du 3 mars 2005, Série C., nº 121, para 103.

25 Affaire de la compétence en matière de pêcheries (Royaume-Uni c. Islande), fond, arrêt, CIJ Rec. 1974, 3, 26, para 59.

26 M. Jones, The Retroactive Effect of the Recognition of States and Governments, 16 British Yearbook of International Law (1935), 42 [notre traduction]. 
ments de définition sont ici d'une importance notable : le fait, le consentement au fait et l'opposabilité du fait.

Lorsque M. Jones évoque une "situation ", d'autres mentionnent un " fait $»^{27}$ ou une $~ s$ situation de fait ${ }^{28}$ La première pierre d'achoppement de la question de la reconnaissance apparaît ici immédiatement : qui établit le fait soumis à reconnaissance ? Posons très simplement l'exemple mentionné de la reconnaissance d'une responsabilité internationale. Dans ce cas, un État établit qu'il y a eu préjudice causé à son endroit. En d'autres termes, l'État supposément lésé établit le fait et demande qu'il soit reconnu. Certains ont ainsi pu laisser entendre que l'établissement objectif du fait était une chimère et que l'objet de la reconnaissance n'était rien de moins qu'une prétention. ${ }^{29}$ De la même manière, dans le cadre de la reconnaissance d'État, ce qui est soumis à la reconnaissance est la revendication de la qualité étatique par les organes mêmes de ce supposé État. ${ }^{30}$ Il serait, par conséquent, plus à propos dans notre définition de parler de proposition de fait plutôt que de fait. ${ }^{31}$

Le deuxième élément saillant de la définition est le consentement à la proposition de fait. Il suppose une action unilatérale de la part d'un sujet typiquement un État - traduisant son acquiescement à la proposition. En d'autres termes, le consentement ne laisse apparaître que l'accord de celui qui l'exprime face à une prétention. Un certain nombre d'auteurs considèrent ainsi que la théorie doit dissocier le consentement à la proposition et par extension, la reconnaissance - de la connaissance du fait situé en

27 J. Charpentier, La reconnaissance internationale et l'évolution du droit des gens, supra note 13, 3 : « [Les objets de la reconnaissance] ont ceci de commun d'être des faits $[\ldots]$ ».

28 Combacau et Sur, Droit international public, supra note 22, 289 : « La reconnaissance est l'acte juridique unilatéral par lequel un État atteste l'existence à son égard d'une situation de fait [...]».

29 Brownlie, supra note 9, 202 : « [Il est d'abord] nécessaire de formuler certaines questions quant à "l'ambition factuelle" de l'entité en question avant [...] [d'envisager] les conséquences juridiques auxquelles l'entité peut aspirer » [notre traduction].

30 A. Jolicoeur, De la reconnaissance en droit international, 6 (2) Les Cahiers de droit (1965), 85, 86 : «La reconnaissance juridique d'un État doit d'abord être faite par la communauté qui la compose ».

31 J. F. Williams, La doctrine de la reconnaissance en droit international et ses développements récents, 44 R.C.A.D.I. (1933), 199, 206 : « La reconnaissance [...] est la réponse favorable à une prétention de posséder un certain caractère, un certain "status" »; F. Münch, supra note 12, 158 : «[...] [L]a reconnaissance [...] signifie le consentement à une proposition, son acceptation comme base de la discussion ultérieure et du comportement futur ». 
amont de la proposition..$^{32}$ En effet, nous l'avons laissé entendre, le fait proposé peut être plus ou moins conforme au fait réel puisque soumis à la subjectivité de celui qui aspire à sa reconnaissance. Mais, le fait proposé pourrait tout aussi bien correspondre à la réalité, cependant que tout consentement lui serait dénié. ${ }^{33}$ La connaissance ontologique du fait n'est, par conséquent, pas la reconnaissance ; laquelle n'est que le consentement libre à la proposition de fait. ${ }^{34}$

Le dernier élément de la définition est l'opposabilité. ${ }^{35}$ Par la reconnaissance de la proposition de fait, le sujet "s'engage à [...] tirer les conséquences que le droit attache » au fait reconnu. ${ }^{36}$ Aussi, en reprenant un des exemples susmentionnés, l'État qui reconnaît sa responsabilité pour le préjudice subi par un autre État peut-il s'en voir opposer les implications juridiques $; 3$ typiquement une obligation de réparation. Cette opposabilité repose sur le principe fondamental de volonté, que la reconnaissance - en tant que mode de formation volontaire du droit ${ }^{38}$ - partage avec la tech-

32 Brownlie, supra note 9, 204.

33 Bierzaneck, supra note $5,123:$ « [...] [N] ous sommes en présence de discordances flagrantes entre ce qui est reconnu et ce qui existe en réalité ». Ce constat a pu trouver une illustration concrète dans le cas de la Rhodésie du Sud. Voir à cet égard Brownlie, supra note 9, 204 : «Dans [le] cas [de la Rhodésie du Sud,] c'est le statut, et non pas la réalité, qui est refusé » [notre traduction].

34 Williams, supra note 31, 215-216. L'auteur y présente un exemple particulièrement éloquent ; celui de la reconnaissance par Louis XIV du fils de Jacques II comme roi d'Angleterre : "Que fit exactement Louis quand il "reconnut" ainsi "Jacques III" ? Évidemment, il n'admettait pas l'existence d'un fait. "Jacques III" [...] n'était pas, en fait, roi d'Angleterre. Louis admettait donc, ou plutôt affirmait, la justesse d'une prétention ; [...] il affirmait que pour lui-même, Louis, subjectivement, l'ancien prétendant était bien roi d'Angleterre. [...] C'était $[\ldots]$ une affirmation de ce que Louis croyait ou prétendait croire ».

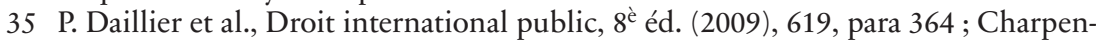
tier, Reconnaissance, supra note 22, para 14 . Certaines théories interrogent néanmoins les rapports entre reconnaissance et opposabilité. Voir p. ex., Verhoeven, Reconnaissance, supra note 3, 6. Les travaux de la Commission de droit international semblent même suggérer que l'opposabilité ne constitue qu'une des conséquences juridiques de la reconnaissance. Voir à ce titre, V. Rodríguez Cedeño, Rapporteur spécial de la CDI sur les actes unilatéraux des États, Sixième Rapport, A/CN.4/534, para 67.

36 Combacau et Sur, supra note 22, 289.

37 Daillier et al., supra note 35, 619, para $364:$ « [...] [Le] sujet [...] accepte que [la] situation [lui] soit opposable [: il] admet que les conséquences juridiques de [celle-ci] s'appliquent à lui ».

38 Ibid., 393, para 234. 
nique conventionnelle. ${ }^{39}$ Le consentement exprimé par l'État le liera juridiquement et aura des effets de droit donnés.

Il serait naturellement impossible d'aborder la question de la reconnaissance sans avoir évoqué le schisme qui n’a pas manqué de diviser la doctrine entre les tenants d'une conception constitutive de l'acte de reconnaissance et les partisans de sa nature et sa portée déclaratives. ${ }^{40}$ En dépit des qualités de chaque vue et de la tentative de synthèse engagée par H. Lauterpacht qui affirme que « la reconnaissance [est] déclaratoire de faits et [...] constitutive de droits ", 41 elles renferment toutes deux leur propre contrariété. L'approche déclarative conçoit l'acte de reconnaissance comme une simple déclaration de la qualité étatique de son objet : la reconnaissance est extérieure à l'État et ne le constitue, ni ne le crée. ${ }^{42}$ En vertu de celle-ci, l'État existe tant et pourvu qu'il réunisse les trois critères prescrits par le droit international : un territoire défini, une population permanente et un gouvernement effectif et indépendant. ${ }^{43}$ Il s'ensuit logiquement que la per-

39 Certains vont, par ailleurs, très loin dans l'analogie entre les deux modes de formation du droit. Voir à ce titre $\mathrm{H}$. Kelsen, Théorie générale du droit international public - Problèmes choisis, 42 R.C.A.D.I. (1932), 117, 278-279 : " La reconnaissance n'est donc rien d'autre qu'une convention [...]. [Elle] est [...] tout simplement la convention introductive à laquelle se rattache la naissance de normes juridiques pour certains sujets, et par conséquent la personnalité juridique réciproque de ces sujets. Et en effet, la réciprocité [...] [est] propr[e] à l'idée de reconnaissance »; A. Lagerwall, Le principe ex injuria non oritur en droit international (2016), 506 : «[L']école critique choisit [...] [d']attribuer à l'acte de reconnaissance une nature conventionnelle qui relie la volonté de l'État revendiquant la reconnaissance d'une situation à la volonté d'un autre État acceptant de lui octroyer cette reconnaissance ".

40 Williams, supra note 31, 206 : «Le point principal d'incertitude - de controverse même $[\ldots]$ repose sur la question de savoir si la reconnaissance d'un [...] État [...] est de caractère constitutif ou simplement déclaratoire [...]».

41 Lauterpacht, Recognition of States, supra note 5, 455 [notre traduction].

42 R. Erich, La naissance et la reconnaissance des États, 13 R.C.A.D.I. (1926), 427, 461 : «Quand un gouvernement étranger reconnaît un nouvel État, il constate, par la même, qu'on se trouve devant un fait, un statut organisé dont l'existence lui paraît incontestable. On le reconnaît parce qu'il existe. On ne le reconnaît pas afin qu'il prenne naissance [...] »; Williams, supra note 31, $207:$ : [La reconnaissance] n'indique pas une opération créatrice d'un fait objectif ».

43 Notre définition s'inspire de certaines positions doctrinales qui font reposer la condition d'indépendance ou de souveraineté - laquelle est fréquemment considérée comme une quatrième condition sine qua non - sur le critère du gouvernement. Voir à cet égard E. Jouannet, Le droit international (2013), 40 : « [...] $[\mathrm{L}]$ 'existence [de l'État] dépend de la réunion d'un certain nombre de faits (terri- 
sonnalité juridique précèderait la reconnaissance, ${ }^{44}$ et que l'État reconnu ou non serait doté des droits et obligations qu'emporte ce statut. ${ }^{45}$ Cependant, si cette approche est pleinement pertinente quant à la suffisance des trois critères originels pour établir le fait-État, leur satisfaction ne suffit pas toujours à faire de celui-ci, comme le démontre la pratique, un État au sens juridique ou un fait juridique-État. ${ }^{46}$ De plus, affirmer que la reconnaissance est un acte purement déclaratoire reviendrait à mettre sérieusement en doute sa nature et ses effets juridiques. ${ }^{47} \mathrm{La}$ conception constitutive, quant à elle, suggère que la proposition de fait-État devient - par le truchement de la reconnaissance agissant comme un prisme - un fait juridiqueÉtat. Exprimée de façon prosaique et poussée jusqu'à sa logique extrême, cette vision signifierait que la reconnaissance ferait entrer l'État «dans le monde du droit $~^{48}$ et qu’à défaut de celle-ci, l'État serait dépourvu de personnalité juridique et de droits. ${ }^{49} \mathrm{La}$ reconnaissance serait ainsi conçue

toire, population, gouvernement effectif et indépendant) dont le droit international ne fait que prendre acte »; B. Stern, La succession d'États, 262 R.C.A.D.I. (1996), 9, 68 : «On sait qu'un État doit posséder trois éléments constitutifs : un territoire, une population et un gouvernement exerçant la souveraineté, aussi bien interne qu'internationale »; J. Verhoeven, La reconnaissance internationale : déclin ou renouveau, 39 Annuaire français de droit international (1993), 7, 37 : « À l'ordinaire, [l'Etat] naît ou meurt de la présence ou de l'absence d'éléments de pur fait : un territoire, une population, un gouvernement indépendant »; F. Poirat, La doctrine des droits fondamentaux de l'État, 16 Droits (1992), 83, 89 : " Population, territoire, gouvernement effectif et indépendant, nous sommes en présence, pour le droit international, des éléments constitutifs de l’État »; H. M. Blix, Contemporary aspects of recognition, 130 R.C.A.D.I. (1970), 587, 622 : « En droit, une entité est généralement réputée être un État si elle possède un territoire, une population, un gouvernement indépendant des autres [...] » [notre traduction] ; Lauterpacht, Recognition of States, supra note 5, $408:$ : [...] [L]es conditions de la qualité étatique telles qu'elles sont établies par le droit international [...] [correspondent à] l'existence d'un gouvernement indépendant exerçant une autorité effective sur une zone définie » [notre traduction].

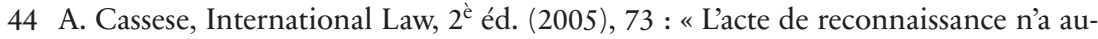
cun effet juridique sur la personnalité internationale de l'entité [...] » [notre traduction].

45 Combacau et Sur, supra note 22, 290.

46 H. Ruiz Fabri, Genèse et disparition de l'État à l'époque contemporaine, 38 Annuaire français de droit international (1992), 153, 163.

47 Kelsen, Recognition, supra note 11, 605-606.

48 C. Santulli, Le statut international de l'ordre juridique étatique. Étude du traitement du droit interne par le droit international (2001), 25.

49 I. Brownlie, supra note 9, 206 : « [...] [Selon] la théorie constitutive, [...] l'acte $[\ldots]$ de reconnaissance $[. .$.$] est une condition préalable à l'existence de droits »$ [notre traduction]. 
comme une condition supplémentaire et nécessaire à l'existence d'un État. ${ }^{50}$ Toutefois, à y regarder de plus près, contrairement aux trois critères mentionnés - lesquels sont objectifs - la reconnaissance est extrinsèque à l'entité étatique. L'État en tant que fait n'est que l'addition de trois conditions factuelles. ${ }^{51}$ La reconnaissance ne devrait, par conséquent, pas être envisagée comme une condition d'existence de l'État.

\section{Reconnaissance de jure et reconnaissance procédurale de l'État: champ de l'étude et problématique}

Nous le voyons, les difficultés de théorisation et de définition de la reconnaissance d'État ressortissent principalement à l'absence de consensus quant à sa nature et à ses effets. Toutefois, dans le cadre de notre étude, l'acte de reconnaissance - parce qu'il se limite à la relation bilatérale entre le sujet étatique en situation de reconnaître et l'entité qui a l'ambition d'être reconnue ${ }^{52}$ - est nécessairement constitutif. ${ }^{53}$ Ainsi définie, la reconnaissance dans le contexte de la situation intersubjective entre États ${ }^{54}$ est à assimiler à la reconnaissance de jure, ${ }^{55}$ notion qui décrit la forme absolue

50 V.-D. Degan, Création et disparation de l'État à la lumière du démembrement de trois fédérations multiethniques en Europe, 279 R.C.A.D.I. (1999), 195, 254-255 : «[Cette approche] prétend que la reconnaissance [...] est [...] le quatrième critère de l'existence même de l'État nouveau ».

51 E. Borchard, Recognition and non-recognition, 36 American Journal of International Law (1942), 108, 110 : «Les indices révélateurs de la qualité étatique sont déterminés et établis par des faits objectifs, non pas par une reconnaissance subjective » [notre traduction].

52 Verhoeven, Droit international public, supra note 7, 78.

53 Cette « reconnaissance individuelle » déploie des effets juridiques constitutifs non pas sur le plan du «droit international général » mais uniquement en « droit international particulier » et dans les droits internes des deux États « à la relation de reconnaissance » ; comme l'explique Wyler, La succession d'États, supra note 19, 1646-1648, paras 43-45 ; voir également Wyler, Théorie et pratique, supra note 8, 267-284 ; Chatelain, La reconnaissance internationale, supra note 2, 720 : « La reconnaissance est l'acte de volonté par lequel chaque État déclare vouloir considérer une autre [entité] [...] comme un autre État. Elle fait ainsi naître [...] l'État reconnu au regard de l'État auteur de la reconnaissance [...]».

54 Combacau et Sur, supra note 22, 290.

55 Notre définition de la reconnaissance de jure n'est pas ici conçue négativement comme le pendant de la reconnaissance de facto, notion controversée et incertaine au regard de la pratique. Voir à ce titre H. Lauterpacht, Recognition in International Law (1947), 329 : "La reconnaissance de facto est une notion relativement insaisissable au regard du droit de la reconnaissance. Il n'existe pas de consensus au- 
que prend l'acte en pratique. ${ }^{56} \mathrm{La}$ reconnaissance de jure produit ainsi la totalité des effets juridiques de la reconnaissance dans sa forme théorisée. ${ }^{57}$ C'est pour cela qu'elle a pu être décrite comme une reconnaissance complète ${ }^{58}$ pleine, ${ }^{59}$ sans réserve, ${ }^{60}$ plénière, ${ }^{61}$ entière ${ }^{62}$ ou encore illimitée. ${ }^{63}$ En d'autres termes, la reconnaissance de jure de la qualité étatique d'une entité lui confere l'ensemble des droits et obligations associés au statut d'État dans sa relation avec l'État qui la reconnaît en tant que tel. ${ }^{64}$

C'est parce que la reconnaissance de jure d'État accordée par l'exécutif interne demeure la référence ${ }^{65}$ qu'il est permis de l'utiliser comme étalon à l'aune duquel sera étudié le mécanisme que nous avons décidé d'appeler, par convention, la reconnaissance procédurale d'État prononcée par le juge interne. La logique exige que cette mise en parallèle se fonde sur un élément d'identité - ou à tout le moins de similarité - entre les deux mécanismes pour qu'elle soit justifiée. Cet élément, nous le postulons, est constitué par la conséquence générale qu'implique la mise en mouvement

tour d'une signification juridique précise. [...] Certains [...] doutent qu'en droit elle puisse être distinguée de la reconnaissance de jure. Ils la considèrent comme une variante politique de celle-ci. Certains l'assimilent à la reconnaissance implicite. D’autres jugent que sa particularité tient au fait qu'elle implique une capacité internationale plus limitée que celle qui découle de la reconnaissance de jure. D'autres la considèrent comme conditionnelle ou provisoire, voire les deux. Certains pensent que sa caractéristique essentielle réside dans son caractère révocable. D'autres encore pensent que ses effets se limitent à l'absence de relations diplomatiques ordinaires ou complètes » [notre traduction].

56 L'identité entre la reconnaissance (non qualifiée ou théorique) et la reconnaissance de jure est attestée par la pratique, dans la mesure où les exécutifs étatiques utilisent les deux expressions comme des synonymes. Voir à ce titre Talmon, supra note $10,91$.

57 Daillier et al., supra note 35, 629.

58 P. M. Brown, Cognition and Recognition, 47 American Journal of International Law (1953) 87, 88.

59 Daillier et al., supra note 35, 629.

60 Brownlie, supra note 9, 207.

61 Institut de droit international, Résolution sur la reconnaissance des nouveaux États et des nouveaux gouvernements, 23 avril 1936, Art. 3.

62 Daillier et al., supra note 35, 629.

63 Brown, supra note 58, 88.

64 Charte de l'Organisation des États américains, 30 avril 1948, Art. 10, 119 RTNU 49, 57 : «La reconnaissance implique l'acceptation, par l'État qui l'accorde, de la personnalité du nouvel État avec tous les droits et devoirs fixés, pour l'un et l'autre, par le droit international ».

65 Jennings, supra note 2, 355 . 
des deux mécanismes : l'octroi ou le refus d'octroi de la qualité étatique et du statut qui en découle.

Le phénomène au centre de l'étude brille par son extension géographique. Les juges français, canadien, allemand, états-unien, néerlandais ou encore japonais se sont en effet prononcés sur la qualité étatique d'une entité au cours de l'instance. L'objectif n'est cependant pas ici de témoigner de l'existence d'une dynamique universelle de reconnaissance par le juge interne, ni d'identifier les politiques jurisprudentielles en la matière propres à chaque système juridictionnel interne, ${ }^{66} \mathrm{ni}$ même encore de procéder à une étude de droit comparé, mais davantage de décrire le phénomène général de la reconnaissance procédurale d'État en ce qu'il présente de commun parmi l'ensemble des affaires identifiées. L'analyse de celles-ci permettra, dans un premier temps, de réfléchir à la nature juridique du mécanisme de la reconnaissance procédurale (II) et d'identifier, dans un second temps, les effets de droit qu'elle implique (III).

\section{Nature et modalités de la reconnaissance procédurale d'État}

Il est permis de décrire l'opération juridique de la reconnaissance procédurale en ayant égard au contexte dans lequel elle se déploie (A) et à la structure qu'elle adopte lorsqu'elle est mise en ouvre (B). Cet essai de définition externe et interne nécessitera toutefois préalablement une description axiomatique, ${ }^{67}$ intensionnelle ${ }^{68}$ et succincte permettant d'identifier l'objet empirique dont il est question. À ce titre et au risque de nous répéter, nous postulons que la (non-)reconnaissance procédurale de l'État est l'opération juridique par laquelle un juge accepte ou refuse d'octroyer la qualité étatique à une entité au terme d'un raisonnement consistant à confronter des faits à la norme d'origine internationale prescrivant les conditions d'existence de l'État.

66 Certains évoquent la difficulté, pour ne pas dire la stérilité, d'une telle approche ; l'un des problèmes tenant à l'absence de cohérence jurisprudentielle interne à chaque État. Voir Verhoeven, Droit international public, supra note 7, 80 ; Verhoeven, Les relations internationales, supra note 2, 26-27. 


\section{A. Le cadre de la reconnaissance procédurale}

Bien que l'observation du contexte dans lequel intervient le mécanisme de la reconnaissance procédurale révèle une indétermination générale de la configuration juridictionnelle et procédurale des différentes affaires pertinentes (1), il apparaît toutefois qu'il n'est mis en mouvement que dans la mesure où le juge parvient à écarter un certain nombre d'obstacles (2).

\section{Une mise en cuvre indifférente aux caractéristiques générales de l'organisation juridictionnelle et de l'instance ${ }^{69}$}

Une appréciation superficielle d'un échantillon restreint mais représentatif d'affaires ${ }^{70}$ montre, tout d'abord, que la summa divisio entre les systèmes juridiques civilistes et ceux basés sur la common law n'est pas déterminante dans la survenance du phénomène. Si bon nombre d'affaires dans lesquelles la reconnaissance procédurale intervient procèdent de juridictions na-

67 L'identification et la description de tout objet d'étude scientifique ne peuvent, par définition, pas être détachées d'axiomes et de postulats posés par le sujet investiguant. L'impossibilité logique d'une objectivité absolue de la démarche scientifique a pu être rendue par la célèbre métaphore d'un philosophe des sciences allemand. Voir à ce sujet O. Neurath, Énoncés protocolaires (1932), in A. Soulez (dir.), Manifeste du Cercle de Vienne et autres écrits : Rudolf Carnap, Hans Hahn, Otto Neurath, Moritz Schlick, Friedrich Waissman (2010), 209, 211 : « Il n’y a aucun moyen qui permettrait de faire, d'énoncés protocolaires dont on se soit définitivement assuré de la pureté, le point de départ des sciences. Il n'y a pas de tabula rasa. Nous sommes tels des navigateurs obligés de reconstruire leur bateau en pleine mer, sans jamais pouvoir le [mettre en cale sèche] [...] ».

68 Intension, in A. Lalande (dir.), Vocabulaire technique et critique de la philosophie, $3^{\text {è } e ́ d . ~(~} 1^{\text {er }}$ tirage 2010, 2013), $528:$ : [...] [L'intension] sert à désigner, au sens le plus large, l'ensemble des caractères représentés par un terme général »; G. Tusseau, Critique d'une métanotion fonctionnelle : La notion (trop) fonctionnelle de notion fonctionnelle, Revue française de droit administratif (2009), 641, 654, para 47 : «Les définitions intensionnelles [...] fonctionnent en indiquant un ensemble de critères au moyen desquels peuvent être sélectionnés des objets, qui rentrent dans la catégorie à définir ».

69 S. Guinchard et al., Droit processuel : Droit commun et droit comparé du procès,

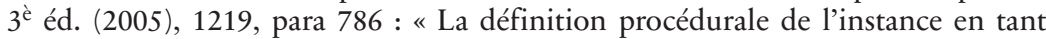
que contenant permet [...] [de la concevoir comme] un mécanisme, déclenché par la saisine de la juridiction, qui s'achève [...] par l'acte de dessaisissement de cette juridiction, [à] savoir la décision [...]».

70 Nous avons pu identifier le mécanisme étudié dans une douzaine d'affaires couvrant une période de 60 ans (1954-2014). 
tionales de tradition romano-germanique, ${ }^{71}$ les cours et tribunaux anglosaxons la pratiquent eux aussi $;^{72}$ tout comme les systèmes mixtes. ${ }^{73} \mathrm{De}$ même, toute juridiction au sein de chaque système peut être amenée à mettre en ouvre l'opération juridique identifiée quelle que soit sa place dans l'organisation juridictionnelle horizontale et verticale de l'État : au plan fédéral ${ }^{74}$ ou de l'entité fédérée $;^{75}$ dans l'ordre judiciaire ${ }^{76}$ ou administratif $;{ }^{77}$ en première instance, ${ }^{78}$ en appel ${ }^{79}$ ou au niveau suprême. ${ }^{80}$

Au-delà de l'organisation juridictionnelle, les caractéristiques de l'instance de chacune de ces affaires sont également étrangères à l'opportunité pour le juge de déclencher le mécanisme. L'observation de trois aspects généraux de la procédure processuelle permet d'illustrer ce constat. S'agissant des différentes phases de l'instance d'abord, la reconnaissance procédurale

71 Tribunal de grande instance de la Seine, Clerget c. Représentation commerciale de la République démocratique du Viêt-Nam, 15 mars 1967, 71 Revue générale de droit international public (1967), 1120 [France]; Tribunal d'arrondissement de La Haye, République démocratique du Timor Oriental, Fretilin et autres. c. Pays-Bas, 21 février 1980, 87 ILR 73 [Pays-Bas] ; Cour suprême de cassation, Italie c. Djukanovic, 28 décembre 2004, ILDC 74 (IT 2004) [Italie].

72 Cour fédérale de district du district de Columbia, Gilmore et autres c. Autorité palestinienne intérimaire autonome et autres, 7 mars 2006, 422 F.Supp.2d 96 (2006) [États-Unis] ; Haute Cour de Justice, République de Somalie c. Woodhouse Drake \& Carey et autres, 13 mars 1992, 94 IRL 608 [Royaume-Uni]. Il importe de souligner que cette dernière décision met en œuvre le mécanisme de reconnaissance procédurale afin de déterminer la qualité gouvernementale d'une entité.

73 Tribunal de district de Tokyo, Limbin Hteik Tin Lat c. Union de Birmanie, 9 juin 1954, 32 ILR 124 [Japon]; Cour supérieure du Québec, Parent et autres c. Singapore Airlines Ltd., 22 octobre 2003, 2033 CanLII 7285 (QC CS) [Canada].

74 Cour d'appel fédérale pour le $1^{\text {er }}$ circuit, Ungar et autres c. Organisation de libération de la Palestine, 31 mars 2005, 402 F.3d 274 (2005) [États-Unis].

75 Parent et autres c. Singapore Airlines Ltd., supra note 73.

76 Cour d'appel de Paris, Strategic Technologies c. Procurement Bureau of the Republic of China - Ministry of National Defence, 30 mars 2011, RG n 10/18825 [France].

77 Tribunal administratif de Cologne, Duché de Sealand, 3 mai 1978, 80 ILR 683 [Allemagne].

78 Gilmore et autres c. Autorité palestinienne intérimaire autonome et autres, supra note 72 .

79 Cour d'appel de Paris, Clerget c. Banque commerciale pour l'Europe du Nord et Banque du commerce extérieur du Vietnam, 7 juin 1969, 74 Revue générale de droit international public (1970), 522 [France].

80 Tribunal fédéral, Wang et consorts c. Office des juges d'instruction fédéraux, 3 mai 2004, ILDC 90 (CH 2004) [Suisse]. 
peut être mise en œuvre aussi bien au stade de la recevabilité, ${ }^{81}$ que lors du traitement au fond de l'affaire. ${ }^{82}$ Quant à la situation processuelle dans laquelle se trouve l'entité faisant l'objet de la mise en ouvre du mécanisme, elle peut être partie (demanderesse ${ }^{83}$ ou défenderesse ${ }^{84}$ ) ou tiers (intervenant forcé, ${ }^{85}$ tiers total ${ }^{86}$ ) à l'instance. S'agissant enfin de la nature du litige $^{87}$ au principal auquel il incombe au juge d'apporter une solution, là encore, l'hétérogénéité est de mise. ${ }^{88}$ Le mécanisme de la reconnaissance procédurale a ainsi pu être mis en mouvement alors que la question juridique au centre de la procédure consistait pour le juge à statuer sur : l'existence d'une immunité pénale au profit du Premier ministre monténégrin, ${ }^{89}$ la nullité de l'assignation introductive d'instance délivrée au défendeur taïwanais, ${ }^{90}$ la perte de la nationalité allemande d'un particulier en raison de l'acquisition de la nationalité sealandaise, ${ }^{91}$ la légalité d'une décision de l'administration suisse d'accorder l'entraide judiciaire à Taïwan ${ }^{92} \mathrm{ou}$ encore le bénéfice de l'immunité de juridiction aux autorités palestiniennes, ${ }^{93}$ etc.

Si le contexte juridictionnel et processuel n'a que très peu d'impact sur l'existence du phénomène, deux constantes peuvent toutefois être relevées à ce stade parmi l'ensemble des affaires évoquées ; lesquelles contribuent à l'unité du phénomène étudié. Premièrement, la qualité étatique de l'entité

81 République démocratique du Timor Oriental, Fretilin et autres. c. Pays-Bas, supra note 71.

82 Ungar et autres c. Organisation de libération de la Palestine, supra note 74.

83 République démocratique du Timor Oriental, Fretilin et autres. c. Pays-Bas, supra note 71.

84 Strategic Technologies c. Procurement Bureau of the Republic of China - Ministry of National Defence, supra note 76.

85 Parent et autres c. Singapore Airlines Ltd., supra note 73.

86 Duché de Sealand, supra note 77.

87 Entendu dans un sens général, commun à l'ensemble des procédures processuelles. Voir à ce titre A. Bolze, La notion de litige juridique, in Études offertes à Jacques Dupichot : Liber Amicorum (2004), 41, 59 : « [Le litige résulte] d'une indétermination du droit applicable à une situation qui appelle l'intervention d'un organe pour y mettre fin par une décision en droit [...]».

$88 \mathrm{La}$ nature hétéroclite des contentieux explique en partie la pluralité des juridictions amenées à appliquer le mécanisme et la diversité des recours y relatifs.

89 Italie c. Djukanovic, supra note 71.

90 Strategic Technologies c. Procurement Bureau of the Republic of China - Ministry of National Defence, supra note 76.

91 Duché de Sealand, supra note 77.

92 Wang et consorts c. Office des juges d'instruction fédéraux, supra note 80.

93 Ungar et autres c. Organisation de libération de la Palestine, supra note 74. 
soumise au test de la reconnaissance procédurale est, par définition, toujours controversée car cette dernière ne bénéficie jamais d'une reconnaissance de jure de la part de l'exécutif de l'État du for. ${ }^{94}$ Deuxièmement, la question de la qualité étatique de l'entité n'est jamais autonome en ce qu'elle ne s'identifie pas à la question litigieuse principale et ne constitue qu'une étape, certes nécessaire, ${ }^{95}$ vers sa résolution.

\section{Le dépassement d'obstacles potentiels à la mise en œuvre du mécanisme}

Il n'est, en principe, pas interdit au juge de se prononcer sur la question de la qualité étatique d'une entité lorsqu'il remplit son office. ${ }^{96}$ Dans les hypothèses où il prend le parti de ne pas y répondre, il s'autolimite d'une certaine façon. ${ }^{97}$ Mais, lorsqu'il décide de juger de la qualité étatique de l'entité, des limites extérieures peuvent s'imposer à lui et influer sur la possibili-

94 Couveinhes-Matsumoto, supra note 17, 282, para 270.

95 Cette condition de nécessité est évidemment largement soumise à la subjectivité du juge. En effet, est ici déjà suggérée l'importance de la liberté de celui-ci dans la mesure où s'il applique le mécanisme de la reconnaissance procédurale, c'est qu'il estime que ce type de raisonnement est non seulement nécessaire mais aussi le plus opportun. Toutefois, un juge peut très bien considérer qu'il y a nécessité de répondre à la question de la qualité étatique d'une entité afin de trancher un litige, en mettant en ouvre un autre test que celui de la reconnaissance procédurale. Il se fondera, en général, sur l'existence ou non d'une reconnaissance de jure de la part de l'exécutif pour en déduire la qualité étatique de l'entité. Voir à ce sujet, une affaire où le juge écarte expressément l'application du mécanisme de reconnaissance procédurale jugé comme inopportun : Cour suprême, Civil Aeronautics Administration c. Singapore Airlines Ltd., 14 janvier 2004, ILDC 86 (SG 2004) [Singapour]. Enfin, le caractère tout à fait relatif de la nécessité est évident lorsqu'un juge refuse de se prononcer sur la qualité étatique d'une entité, tout en statuant tout de même sur le litige au principal ; usant ainsi de stratégies d'évitement. Une de ces stratégies trouve son expression dans la doctrine jurisprudentielle américaine de non justiciability doctrine ou de political question doctrine; bien que le juge américain ait tendance à en réduire le champ. Voir à ce titre, p. ex., Cour fédérale de district du district sud de New York, Sokolow et autres c. Organisation de libération de la Palestine et autres, 30 septembre 2008, 583 F.Supp.2d 451. Si ces deux dernières pratiques judiciaires ne nous intéressent pas directement ici, elles permettent de délimiter négativement le cadre de la reconnaissance procédurale.

96 Verhoeven, Les relations internationales, supra note 2, 29.

97 Voir supra note 95 sur les stratégies d'évitement. C'est d'ailleurs ce qu'a fait la Cour de cassation française dans l'affaire, Strategic Technologies c. Procurement Bureau of the Republic of China - Ministry of National Defence, 19 mars 2014, 
té de mettre en œuvre le mécanisme visé. Si de nombreux commentateurs ont pu suggérer que l'occurrence du phénomène de la reconnaissance procédurale était plus limitée dans les systèmes de common law - précisément en raison de la place du juge anglo-saxon par rapport à l'exécutif ${ }^{98}$ - qu'au sein des juridictions de tradition civiliste, il apparaît toutefois que les données externes qui s'imposent aux deux juges sont comparables bien qu'elles puissent faire l'objet d'un traitement quelque peu différent. ${ }^{99}$ En tout état de cause, c'est la liberté dont se prévalent pleinement les deux juges qui participera de la mise en mouvement du mécanisme. ${ }^{100}$

Parce que l'opération juridique de la reconnaissance procédurale est - nous le verrons plus avant - un mécanisme de qualification juridique des faits, la disponibilité, la place et surtout la valeur probante de ces faits auront une incidence sur la survenance du mécanisme, et ceux-ci pourront même constituer un obstacle à sa mise en ouvre effective. Typiquement, deux éléments factuels - ayant une origine commune en ce qu'ils sont générés par l'exécutif - auront une influence déterminante sur le mécanisme, influence qui sera fonction du traitement que leur réservera le droit de la preuve. Ces deux éléments sont : l'absence de reconnaissance de jure et l'avis gouvernemental. ${ }^{101}$

Premièrement, la non-reconnaissance de la qualité étatique de l'entité par l'exécutif a été mentionnée plus haut comme un des éléments constants du cadre général dans lequel survient la reconnaissance procédurale. Il peut s'agir soit d'une simple absence de reconnaissance, soit d'un re-

119 Revue générale de droit international public (2015), 276. Saisie sur pourvoi, afin d'infirmer la décision de la Cour d'appel de Paris ayant reconnu procéduralement Taïwan (voir supra note 76), la Cour de cassation confirme la décision d'appel en évitant de se prononcer sur la qualité étatique de Taïwan. Certains commentateurs y voient la mise en ouvre d'une "stratégie d'évitement ». Voir à cet égard, le commentaire de la décision par : B. Tranchant, 119 Revue générale de droit international public (2015), 279, 286.

98 J. Crawford, The Creation of States in International Law, $2^{\mathrm{è}}$ éd. (2006), 17 ; Lauterpacht, Recognition in International Law, supra note 55, 70. Voir également Verhoeven, Les relations internationales, supra note 2, 33 : « Il y a[urait] là deux attitudes apparemment contradictoires. [...] Sommairement rapportée, la pratique anglo-saxonne de soumission à l'exécutif contraste en effet avec l'indépendance totale dont jouit en principe le juge dans les pays de civil law » [nous soulignons].

99 Verhoeven, Les relations internationales, supra note 2, 35 .

100 Ibid., 37 : "Qu'il soit de common law ou de civil law, le juge est souverain dans l'appréciation du droit où se manifeste pleinement son indépendance. Il n'y a de ce point de vue aucune différence de principe entre les deux systèmes ».

101 La question de leur admissibilité en preuve est, en général, toujours acquise. 
fus exprès de reconnaître. Dans tous les cas, cette circonstance ne peut évidemment pas être ignorée par le juge, ${ }^{102}$ lequel ne manque que rarement de la relever. Il est patent, à ce titre, que les juridictions continentales et mixtes refusent en général d'accorder toute force probante à celle-ci. ${ }^{103}$ Tout au plus, elles lui conferent une valeur peu concluante ou indicative. ${ }^{104}$ La situation est plus complexe en ce qui concerne les juridictions de common law. Rares sont les cours qui, comme la Cour suprême singapourienne, attachent pleine force probante à l'absence de reconnaissance de jure, ${ }^{105}$ rendant logiquement superflue la mise en œuvre du mécanisme de reconnaissance procédurale. Aux États-Unis, il est plus difficile de dresser un tableau cohérent de la valeur probante qu'accordent les juges à la non reconnaissance de jure. Si certains juges suggèrent, de manière obscure, " qu'il pourrait être soutenu qu'un État étranger [...] est une entité qui a été reconnue comme souveraine par le gouvernement des États-Unis », ${ }^{106}$ et appliquent par la suite, sans grande conviction, le mécanisme de la reconnaissance procédurale ; ${ }^{107}$ d'autres ne mentionnent même pas l'absence de reconnaissance de la part de l'exécutif - lui déniant a fortiori toute perti-

102 Clerget c. Banque commerciale pour l'Europe du Nord et Banque du commerce extérieur du Vietnam, supra note 79, 524 ; Strategic Technologies c. Procurement Bureau of the Republic of China - Ministry of National Defence, supra note 76, 4.

103 Italie c. Djukanovic, supra note 71, para 14 : «Dans le cas présent, il est tout à fait indifférent que l'Italie (comme d'autres États) ait reconnu ou non le Monténégro en tant qu'État indépendant » [notre traduction, nous soulignons].

104 République démocratique du Timor Oriental, Fretilin et autres. c. Pays-Bas, supra note 71, 74, para 3 : «S'agissant de l'existence de la République [du Timor Oriental] en tant qu'État, les parties conviennent que le fait que les Pays-Bas et pratiquement l'ensemble de la communauté internationale ne reconnaissent pas la République n'est pas déterminant. La Cour partage l'opinion des parties [...] » [notre traduction, nous soulignons]. Si dans certains systèmes, les juridictions ne semblent pas expliciter la valeur probante accordée à la non-reconnaissance de jure, leur motivation laisse peu de doute quant à la place que celle-ci occupe dans leur raisonnement. Voir Parent et autres c. Singapore Airlines Ltd., supra note 73, para 55 : «La reconnaissance d'un État par les autres États ne crée pas l'État [...] »; Limbin Hteik Tin Lat c. Union de Birmanie, supra note 73, 124 : « [...] [L']Union [de Birmanie] doit être reconnu en tant qu'État étranger [...] même si le Japon ne l'a pas reconnue " [notre traduction].

105 Voir Civil Aeronautics Administration c. Singapore Airlines Ltd., supra note 95, paras 25, 31 .

106 Ungar et autres c. Organisation de libération de la Palestine, supra note 74, para 4 [notre traduction].

107 Ibid. Cette décision a été rendue en appel et confirme la décision attaquée (Cour fédérale de district du district de Rhode Island, Ungar et autres c. Autorité pales- 
nence et valeur probante dans la question de la détermination de la qualité étatique. ${ }^{108} \mathrm{Il}$ apparaît toutefois que lorsque l'exécutif « s'oppose catégoriquement à l'idée de [voir] en [l'entité] un [État] souverain » ${ }^{109}-$ et non pas, lorsqu'il s'est simplement abstenu de reconnaître, ${ }^{110}$ - le juge y voit un élément à forte valeur probante que le test de la reconnaissance procédurale ne pourrait renverser. ${ }^{111}$

La logique qui a cours dans la détermination de la valeur probante de la décision de l'exécutif de ne pas reconnaître de jure l'entité se retrouve peu ou prou dans le traitement réservé aux avis ${ }^{112}$ de l'exécutif. Ceux-ci sont des actes de procédure - car émis spécifiquement dans le cadre de l'instance -, par un organe dépendant de l'exécutif ou appartenant à celui-ci, ${ }^{113}$ exprimant une opinion s'agissant d'une question qui, dans notre cas, corres-

tinienne et autres, 23 avril 2004, 315 F.Supp.2d 164 (D.R.I. 2004)). Toutefois, le ratio decidendi du jugement de première instance défie, à notre avis, toute logique juridique. Le juge met préalablement en œuvre notre méthode de détermination de la qualité étatique d'une entité (test qui s'avère non concluant) pour en refuser finalement toute portée décisive en raison de l'absence de reconnaissance de jure de la part de l'exécutif états-unien : «[...] [L]a Palestine ne satisfait pas aux quatre critères constitutifs de la qualité étatique et n'est pas un État au regard des principes juridiques internationaux en vigueur. [...] Même à supposer [...] que l'État de Palestine existe, l'[Autorité palestinienne] n'est pas en droit de bénéficier de l'immunité [de juridiction] car rien ne prouve que les États-Unis aient reconnu [...] la Palestine en tant qu'État souverain » [notre traduction].

108 Gilmore et autres c. Autorité palestinienne intérimaire autonome et autres, supra note 72 .

109 Ungar et autres c. Autorité palestinienne et autres, supra note 107, [notre traduction].

110 Wyler, Théorie et pratique, supra note 8, 183, para $33:$ : [...] [U]ne distinction doit être faite entre refus exprès de reconnaissance [...] et absence de décision en la matière $[. .$.$] ».$

111 Cette position a pu être expliquée par la propension du juge de common law à vouloir parler « d'une seule et même voix » avec l'exécutif. Voir ibid., 181, para 31. Toutefois, il a pu être dit que le fait pour le juge de conférer une valeur probante à une (non-)reconnaissance par l'exécutif lorsque celle-ci est fondée sur des motivations politiques peut entamer la logique juridique et le bien-fondé de sa décision. Voir à ce sujet, Affaire Tinoco (Royaume-Uni c. Costa Rica), 18 octobre 1923, 1 RIAA 369, 381 ; Brownlie, supra note 9, 200.

112 Le nominalisme n'est pas de mise. Chaque droit interne use de son propre vocabulaire en la matière : avis en France, certificat au Canada, statement ou certificate au Royaume-Uni et aux États-Unis, nota en Italie, etc.

113 Nous ne faisons pas de différence ici entre l'avis du Ministère public - lequel est partie à l'instance (voir Loïc Cadiet et al., Théorie générale du procès, $1^{\mathrm{è}}$ éd. (2010), 715-717) - et l'avis produit par l'administration ministérielle (laquelle est tiers à l'instance). 
pond à celle de la qualité étatique d'une entité. Bien que les modalités d'édiction de ces avis different selon les pays, ${ }^{114}$ dans toutes les juridictions, lorsqu'un avis est un émis par l'exécutif, celui-ci constitue un élément de preuve important dont il doit obligatoirement être tenu compte. ${ }^{115} A$ contrario, l'absence d'avis prive le juge d'un élément de preuve à haute valeur probante, mais l'autorise ainsi à mettre en œuvre le mécanisme de reconnaissance procédurale, mécanisme qui mobilisera des éléments de preuve qui, eux, sont disponibles. ${ }^{116} \mathrm{Si}$ un avis est communiqué au juge et aux parties, il appartient au juge de déterminer sa place par rapport aux autres faits et de l'interpréter afin de lui attacher une certaine valeur probante. Dans certaines affaires, un avis exprimé en termes sibyllins a pu être reproduit dans la décision sans que le juge n'ait semblé ni l'interpréter, ni en tenir compte dans la détermination de la qualité étatique de Taïwan ; ${ }^{117}$ suggérant la faible valeur probante de cet élément. Toutefois, il apparaît généralement que lorsque l'avis est interprété par le juge comme clair, alors il constitue un élément, certes probant, mais non pas déterminant pour

114 Là où la procédure est accusatoire, il incombe, en général, aux parties de saisir le ministère des affaires étrangères d'une demande d'avis quant à la qualité étatique d'une entité donnée. Voir République de Somalie c. Woodhouse Drake \& Carey et autres, supra note 72, 618 : «En [...] l'espèce, le ministère des affaires étrangères et du Commonwealth a répondu à trois reprises aux demandes formulées par les avocats des parties [...]» [notre traduction] ; Parent et autres c. Singapore Airlines Ltd., supra note 73, paras 21-22 : "La preuve de [la défenderesse en garantie] ne comporte pas de certificat [...]. Le Tribunal ignore si [elle] a tenté d'obtenir ou non un tel certificat. De son côté, [...] la partie adverse, a communiqué avec le ministère [des affaires étrangères et du commerce international] à ce sujet. En réponse à sa demande, [il] a indiqué ne pas pouvoir y répondre positivement et $[\ldots]$ qu'aucun certificat ne serait émis [...] ». Dans les autres systèmes, l'affaire est, en principe, simplement communiquée par la juridiction au ministère pour lui donner la possibilité de s'exprimer. Mais le juge peut également, de manière plus formelle, requérir directement un avis auprès de celui-ci. Voir Italie c. Djukanovic, supra note 71.

115 Il apparaît que, dans de nombreux systèmes, notamment de common law, l'avis émis est considéré comme contraignant. Toutefois, il appartient au juge de l'interpréter souverainement. Voir à ce sujet Crawford, The Creation of States in International Law, supra note 98, 17 ; Parent et autres c. Singapore Airlines Ltd., supra note 73, para 49 : « [Le certificat] dépos[é] au dossier étant concluan[t], le tribunal est alors lié par le contenu sous réserve toutefois de l'interpréter ».

116 Parent et autres c. Singapore Airlines Ltd., supra note 73 ; Ungar et autres c. Organisation de libération de la Palestine, supra note 74, para 2.

117 Strategic Technologies c. Procurement Bureau of the Republic of China - Ministry of National Defence, supra note 76, 4 . 
conclure à l'existence ou à l'absence de qualité étatique ; révélant ainsi sa place parmi les autres éléments factuels. ${ }^{118}$

Au-delà de l'apparente disparité du traitement réservé à ces deux types d'éléments de fait portés ${ }^{119}$ devant le juge, il apparaît que ceux-ci, lorsqu'ils ne sont pas absents dans les affaires identifiées, ne sont jamais considérés comme des preuves parfaites. L'indisponibilité de faits concluants - appréciation qui révèle la pleine liberté du juge - permet ainsi à celui-ci de justifier du recours au mécanisme de la reconnaissance procédurale.

\section{B. La mise en ouvre de la reconnaissance procédurale}

La mise en œuvre de l'opération juridique de la reconnaissance procédurale par le juge suit la logique syllogistique du raisonnement judiciaire ${ }^{120}(1)$. Celui-ci a, en effet, recours à la méthode de la confrontation des faits au

118 Ainsi, dans deux affaires, l'exécutif a émis des avis reposant sur des éléments factuels et concluant à l'absence de qualité étatique et de qualité gouvernementale des entités concernées ; ce qui n’a pas empêché le juge d'appliquer le test de la reconnaissance procédurale. Voir respectivement, Italie c. Djukanovic, supra note 71, paras 17, 26 ; République de Somalie c. Woodhouse Drake \& Carey et autres, supra note 72, 619: «[...] [Il est] question pour la Cour [...] d'apprécier elle-même les preuves, de formuler des conclusions de fait sur la base de toutes les preuves pertinentes présentées devant elle et d'en tirer la conclusion juridique qui s'impose [...]. [Les avis] du ministère des affaires étrangères et du Commonwealth ne constituent qu'une part des preuves dans cette affaire » [notre traduction].

119 Et, c'est bien en sens qu'ils peuvent être des limites potentielles à la mise en œuvre de la reconnaissance procédurale. Contrairement aux faits mobilisés dans l'opération juridique étudiée - lesquels sont, nous le verrons, produits par le juge lui-même agissant comme un juge d'instruction -, l'avis gouvernemental et la non-reconnaissance de jure sont des faits reçus par le juge et qu'il ne peut ignorer. Nous pouvons, à ce titre, rapprocher ce constat de celui de L. Cadiet et E. Jeuland, Droit judiciaire privé, 8 è éd. (2013), 429, para 544 : "Il est clair que le juge n'est [pas seulement] celui qui, passivement, reçoit le fait [...] ; il est [...] celui qui, de manière active, peut prendre lui-même le fait $[\ldots]$ ».

120 Sur les critiques tendant à considérer le raisonnement juridictionnel comme ne se réduisant pas à une formulation de propositions syllogistiques, voir C. Perelman, Logique juridique : Nouvelle rhétorique, $2^{\mathrm{e}}$ éd. (1979), paras 2-3, 98. Voir également E. Jeuland, Syllogisme judiciaire, in L. Cadiet (dir.), Dictionnaire de la justice (2004), 1269. 
droit $^{121}$ : il choisit la norme applicable, établit les faits pertinents de l'espèce et les qualifie juridiquement. ${ }^{122}$ Cette démarche trahit la prépondérance du fait dans la reconnaissance procédurale, une caractéristique qui autorise à s’interroger sur la véritable nature du mécanisme étudié (2).

\section{Le triomphe du raisonnement logico-déductif ${ }^{123}$}

L'analyse de l'ensemble des décisions pertinentes nous convainc que la reconnaissance procédurale est le résultat de l'application de la méthode syllogistique $;{ }^{124}$ laquelle est présente à un niveau ou à un autre dans le raisonnement de tout juge quel que soit le système dans lequel il officie. ${ }^{125} \mathrm{Au}-$ delà, il peut être dit que la nature de la reconnaissance procédurale se confond avec l'office même du juge, lequel doit, en définitive, toujours faire droit à une prétention en la reconnaissant. Illustrons ce constat en posant un litige dans lequel le demandeur, victime d'un accident de la route, souhaiterait établir la responsabilité délictuelle du défendeur, chauffeur du véhicule à l'origine de l'accident. Il est, par conséquent, demandé au juge par le plaignant de reconnaître une proposition de fait. Sa prétention, sa proposition de fait est qu'il a été lésé, qu'il a subi un préjudice - et accessoirement, que la responsabilité du défendeur est engagée et qu'il est en droit d'obtenir réparation. Afin de faire droit ou non à sa demande, le juge ne pourra pas se dispenser de l'établissement du fait au-delà de la proposition de fait. Il aura à confronter la norme - sur laquelle se fonde la proposition de fait - aux faits. Très sommairement, s'il y a identité entre la proposition de

121 J.-L. Bergel, Méthodologie juridique (2001), 361 ; Alexandrowicz-Alexander, The Quasi-Judicial Function in Recognition of States and Governments, supra note $11,632$.

122 En pratique, il est toutefois difficile de discerner - aussi bien dans l'opération intellectuelle du juge que dans le raisonnement tel qu'il apparaît dans la motivation - les contours de chaque étape et leur chronologie véritable.

123 Voir à ce sujet la synthèse tout à fait pertinente de la question par P. Deumier, Présentation, in P. Deumier (dir.), Le raisonnement juridique : Recherche sur les travaux préparatoires des arrêts (2013), 1, 1-3.

124 La logique syllogistique dans l'application du droit par le juge peut être rendue de la manière suivante : "La conclusion, exprimée dans [...] la décision de justice, $s[\mathrm{e}]$ déduit du rapprochement [de deux] prémisses : la majeure, constituée par l'énoncé de la règle de droit, et la mineure consistant dans la constatation des faits de l'espèce». Voir J.-L. Bergel, Méthodologie juridique, supra note 121, 362.

125 Sur une critique de l'absence d'universalité du raisonnement judiciaire syllogistique, voir Jeuland, Syllogisme judiciaire, supra note 120, 1272. 
fait et le résultat de la qualification juridique des faits établis, le juge reconnaîtra la première. Ainsi relativement à cette simulation de litige, le juge devra établir le(s) fait(s) suivants : le préjudice de la victime est dû à l'accident de la route, le défendeur est le chauffeur du véhicule, l'accident a été causé par la faute du chauffeur, etc. Le juge s'évertuera ensuite à confronter ces faits à la norme, laquelle prévoit les conditions à satisfaire pour que soit reconnue sa prétention (la responsabilité délictuelle et le droit à indemnisation). Dans le cadre de la reconnaissance procédurale, la logique est strictement identique.

Nous nous proposons, dans cette perspective, d'analyser le raisonnement adopté par le juge dans deux ${ }^{126}$ des affaires identifiées. Ce choix se justifie dans la mesure où, au regard de plusieurs éléments de fait et de droit, ${ }^{127}$ ces deux décisions mettant en jeu l'opération juridique de la reconnaissance procédurale se situent aux deux extrémités d'un spectre qui rassemble l'ensemble de la douzaine d'affaires évoquées depuis le début de notre contribution.

La première affaire qui nous intéresse est celle dont a eu connaître une juridiction canadienne, relative à la détermination du statut étatique de Taïwan ${ }^{128}$ : Parent et autres c. Singapore Airlines. ${ }^{129}$ En l'espèce, le requérant, citoyen canadien, emprunte le 31 octobre 2000 un vol de la compagnie aérienne Singapore Airlines entre Singapour et Montréal, vol qui effectue une escale à Taipei, sur l'île de Taïwan. Lors du décollage depuis l'aéroport international de Taipei, l'appareil s'écrase faisant 81 morts et un blessé ; François Parent. Celui-ci introduit un recours en responsabilité contre la compagnie aérienne. Singapore Airlines réfute sa responsabilité et engage, à son tour, celle du gestionnaire et exploitant de l'aéroport : l'Administration de l'aviation civile (AAC) du ministère des transports de la République de Chine. ${ }^{130}$ En réponse, l'AAC invoque l'immunité de juridiction dont bénéficie tout État devant les juridictions étrangères. Singapore Air-

126 Parent et autres c. Singapore Airlines Ltd., supra note 73 ; Duché de Sealand, supra note 77.

127 Ces éléments distinctifs - lesquels ressortissent à l'entité faisant l'objet de l'opération juridique visée, au résultat de la mise en œuvre du mécanisme, au litige au principal, au contexte juridictionnel et de l'instance, aux éléments saillants du raisonnement judiciaire - seront révélés dans les développements dédiés à chaque espèce.

128 Voir à ce sujet un commentaire succinct de l'affaire par P. L. Hsieh, An Unrecognized State in Foreign and International Courts: the Case of the Republic of China on Taiwan, 28 Michigan Journal of International Law (2008), 765, 790-791.

129 Supra note 73.

130 La République de Chine est la dénomination officielle de Taïwan. 
lines conteste cet argument car, de son avis, Taïwan n'est pas un État. Ainsi, la question incidente, nécessaire à la résolution du litige au principal, était de savoir si l'AAC bénéficiait de l'immunité de juridiction.

Cette question procédait de la proposition de fait défendue par l'AAC, au nom de l'exécutif taïwanais, selon laquelle Taïwan était doté de la qualité étatique. Pour confirmer ou infirmer cette prétention, la Cour supérieure du Québec emploie le raisonnement décrit ci-dessus. Elle établit d'abord les faits. ${ }^{131}$ En l'espèce, les éléments factuels les plus importants établis par la Cour sont les suivants : le gouvernement taïwanais est propriétaire de l'aéroport international de Taipei ; l'AAC est l'opérateur de l'aéroport international de Taipei et un département du ministère des transports et des communications du gouvernement de Taïwan sans existence juridique distincte de ce ministère ; le Canada reconnaît la validité d'un passeport émis par les autorités de Taïwan ; Taïwan n'est pas reconnu comme État par le gouvernement canadien; le ministère des affaires étrangères canadien n’a pas émis de certificat au cours de la procédure. Et surtout, la Cour déclare les faits suivants comme incontestables ${ }^{132}$ : l'île de Taïwan constitue un territoire défini d'une surface de $36006 \mathrm{~km}^{2}$; l'île de Taïwan est occupée par une population permanente de 22,5 millions d'habitants ; un gouvernement effectif existe sur l'île de Taïwan, il s'agit d'une démocratie parlementaire avec chef d'État et Premier ministre ; le gouvernement de Taïwan entre en relation avec d'autres États.

La Cour procède, en second lieu, à une qualification juridique de ces faits. ${ }^{133}$ Les faits établis vont être confrontés à la norme qui prescrit les conditions d'existence de l'État. Cette norme, pour laquelle le juge opte, est d'origine internationale ${ }^{134}$ et peut être formulée sous la forme d'une "proposition hypothétique ${ }^{135}$ simplifiée - laquelle commande un raison-

131 Encore une fois, il est difficile de croire que l'établissement des faits - phase qui, dans le cas d'espèce, apparaît la première dans la motivation du juge - précède véritablement en pratique l'identification par le juge de la norme applicable.

132 Parent et autres c. Singapore Airlines Ltd., supra note 73, para 20.

133 Elle peut être conçue, en termes généraux, comme une « opération intellectuelle [...] juridique [...] consistant à prendre en considération l'élément qu'il s'agit de qualifier [...] et à le faire entrer dans une catégorie juridique[,] d'où résulte, par rattachement, le régime juridique qui lui est applicable ». Voir Qualification, in G. Cornu (dir.), Vocabulaire juridique, 10 è éd. (2014), 835.

134 Fréquemment, la norme que cherche à appliquer le juge provient d'une source écrite de droit interne qui contient en son sein l'énoncé de la qualité "État ", mais qui ne la définit pas. Le juge recherchera donc, dans le droit international, la définition à appliquer.

135 Expression empruntée à la typologie de Santulli, supra note 48, 22-25. 
nement syllogistique - selon laquelle : si l'entité a un territoire défini, une population permanente et un gouvernement effectif et indépendant, alors l'entité est un État. Aussi le juge commence-t-il par se fonder sur l'énoncé de la norme codifiée dans la Convention de Montevideo de 1933, ${ }^{136}$ pour y confronter les faits pertinents et conclure par syllogisme à la satisfaction par l'entité taïwanaise des conditions factuelles faisant d'elle un État. Il parvient donc à la décision - après avoir vérifié que le résultat de la qualification juridique des faits correspondait à la proposition de fait - que, Taïwan étant un État, il est en droit de bénéficier de l'immunité de juridiction.

La deuxième affaire qui offre matière à réflexion, malgré son caractère pour le moins surréaliste, est celle dont a eu à connaître une juridiction administrative allemande. ${ }^{137}$ Le plaignant, allemand de naissance, s'adresse, en premier lieu, à l'administration allemande afin qu'elle reconnaisse sa nouvelle nationalité, récemment obtenue de la Principauté de Sealand. ${ }^{138}$ En effet, selon une disposition du droit interne allemand, un individu qui n'est ni domicilié, ni résident permanent en Allemagne perd la nationalité allemande s'il acquiert une nationalité étrangère. Toutefois, l'administration notifie au plaignant qu'il n'a pas perdu sa nationalité allemande car la Principauté de Sealand ne constitue pas un État étranger. Le plaignant demande ainsi au juge d'annuler la décision administrative et de la réformer. Le Tribunal administratif de Cologne confirme la décision : «Étant donné que la [...] Principauté de Sealand ne constitue pas un État au sens du droit international, le plaignant n'a pas acquis de nationalité étrangère ».139 Le raisonnement syllogistique adopté par le Tribunal pour parvenir à cette

136 Convention sur les droits et devoirs des États, 26 décembre 1933, Art. 1, 165 RTSDN 19, 24 : "L'État comme personne de droit international doit réunir les conditions suivantes : une population permanente, un territoire déterminé, un gouvernement, la capacité d'entrer en relation avec d'autres États ». La Convention, on le voit, énonce une quatrième condition d'existence de l'État. Cette capacité d'entrer en relation avec d'autres États est fréquemment conçue par la doctrine comme le critère d'indépendance que certains juristes rattachent directement au troisième critère (celui du gouvernement effectif et indépendant, voir supra note 43). La motivation de la décision confirme bien que le juge canadien conçoit ce critère comme réductible à l'indépendance du gouvernement taïwanais.

137 Duché de Sealand, supra note 77.

138 La Principauté de Sealand est une ancienne plateforme militaire britannique en Mer du Nord, datant de la seconde guerre mondiale. Après son abandon par les autorités, Roy Bates, ancien major de l'armée britannique, s'en empare. L'indépendance de la principauté est proclamée le 2 septembre 1967. La plateforme possède une surface de $550 \mathrm{~m}^{2}$ et compte 4 à 28 habitants selon l'époque.

139 Duché de Sealand, supra note 77, 685 [notre traduction]. 
conclusion est identique à celui mis en évidence dans la décision précédente. ${ }^{140} \mathrm{Il}$ vérifie si la proposition de fait dont le plaignant souhaite obtenir la reconnaissance - sa nationalité sealandaise et, en amont, la qualité étatique de la Principauté - est juridiquement avérée par la confrontation de la norme aux faits. En élaborant une motivation très fouillée et largement appuyée sur la doctrine, le juge allemand caractérise l'absence d'au moins deux des trois conditions indispensables à la qualité étatique de la Principauté de Sealand : le territoire déterminé et la population permanente. ${ }^{141}$

La portée n'est pas anecdotique. La décision concluant à l'absence de qualité étatique de l'entité a pour corollaire l'inexistence des conséquences juridiques découlant normalement du statut d'État, notamment toutes celles se rapportant au concept de nationalité. Cette position privilégiant les faits et la norme internationale constitue bien la mise en ouvre du mécanisme de reconnaissance procédurale, bien que le test aboutisse, en réalité, à un résultat de non-reconnaissance procédurale. ${ }^{142}$

\section{Fait, effectivité et reconnaissance}

L'étude de l'ensemble des jurisprudences internes où l'opération juridique de la reconnaissance procédurale est menée commande un constat unique mais essentiel : le seul dénominateur commun est la force conférée à l'éta-

140 Raisonnement commun à l'ensemble des décisions identifiées dans notre contribution comme mettant en œuvre le mécanisme de reconnaissance procédurale.

141 Dans la mesure où les conditions d'existence de l'État en vertu du droit international sont cumulatives, de nombreuses juridictions ne mènent pas le test jusqu'à son terme lorsqu'une des conditions n'est pas satisfaite. Voir à ce titre, Gilmore et autres c. Autorité palestinienne intérimaire autonome et autres, supra note 72 , para 9 : «Compte tenu de [...] l'absence d'un des critères [nécessaires] à la qualité étatique, il n'est pas indispensable pour la Cour d'examiner [plus avant la satisfaction des autres critères] » [notre traduction].

142 L'objectif n'est évidemment pas de décrire avec précision toutes les subtilités du raisonnement de l'ensemble des affaires où le mécanisme de reconnaissance procédurale est mis en mouvement, mais d'identifier, comme nous venons de le faire, la logique générale et commune du raisonnement du juge en la matière. Il convient, à ce titre, de souligner que la motivation du juge n'est pas toujours aussi étoffée que pour les deux affaires en vedette. Parfois, le juge n'avance pas les faits établis dans sa motivation. Dans d'autres cas, le test de la reconnaissance procédurale n'est pas mené in extenso. Parfois, encore, nous l'avons vu, la norme de référence contient quatre conditions cumulatives, voire n'est pas posée. Ces différences minimes dans la motivation du juge n'entament toutefois pas l'unité d'ensemble du phénomène étudié. 
blissement du fait. Aussi le mécanisme mis en œuvre débouchera-t-il sur une reconnaissance procédurale de l'entité réclamant la qualité d'État, si les faits le corroborent. ${ }^{143}$ Au contraire, le mécanisme aboutira à une non-reconnaissance procédurale pour une entité revendiquant cette qualité, si les faits l'infirment. ${ }^{144} \mathrm{Ce}$ raisonnement a une conséquence logique : au regard des faits établis, le juge est en quelque sorte en situation de "compétence liée $»{ }^{145}$ Mais surtout, le mécanisme de reconnaissance procédurale constitue l'application pratique de l'idée prescriptive que se font certains théoriciens de la reconnaissance selon laquelle celle-ci devrait s'intéresser aux faits. ${ }^{146}$

Il est, en outre, permis de suggérer que la mise en ouvre de la reconnaissance procédurale, en conférant une place centrale à la norme provenant de

143 L'Union de Birmanie a été reconnue procéduralement dans l'affaire : Limbin Hteik Tin Lat c. Union de Birmanie, supra note 73. Le Viêt-Nam du Nord a été reconnu procéduralement dans deux affaires : Clerget c. Représentation commerciale de la République démocratique du Viêt-Nam, supra note 71 ; Clerget c. Banque commerciale pour l'Europe du Nord et Banque du commerce extérieur du Vietnam, supra note 79. Taïwan a été reconnu procéduralement dans de nombreuses affaires : Parent et autres c. Singapore Airlines Ltd., supra note 73 ; Wang et consorts c. Office des juges d'instruction fédéraux, supra note 80 ; Strategic Technologies c. Procurement Bureau of the Republic of China - Ministry of National Defence, supra note 76.

144 La principauté de Sealand n'a pas été reconnue procéduralement dans : Duché de Sealand, supra note 77. Le Timor oriental n'a pas été reconnu procéduralement (à la date de l'affaire, en 1980, l'entité était encore sous occupation indonésienne et ne constituait donc pas un État indépendant) : République démocratique du Timor Oriental, Fretilin et autres. c. Pays-Bas, supra note 71. Le Monténégro n'a pas été reconnu procéduralement (à la date de l'affaire, en 2005, l'entité était encore une république constitutive de l'État fédéral de Serbie-et-Monténégro et ne constituait donc pas un État indépendant) : Italie c. Djukanovic, supra note 71. La Palestine n'a pas été reconnue procéduralement dans plusieurs affaires : Ungar et autres c. Organisation de libération de la Palestine, supra note 74 ; Gilmore et autres c. Autorité palestinienne intérimaire autonome et autres, supra note 72 .

145 Daillier et al., supra note 35, 624, para 367 : « Il peut paraître étonnant que [...] la compétence pour reconnaître un État [...] ne soit pas une compétence liée [...] puisque l'État est une réalité objective [...] »; Alexandrowicz-Alexander, The Quasi-Judicial Function in Recognition of States and Governments, supra note 11, 632 : «Un juge n'exerce pas de pouvoir discrétionnaire [...] ; il applique une règle de droit aux faits de l'espèce " [notre traduction].

146 Lauterpacht, Recognition of States, supra note 5, 385 : «Reconnaitre une communauté en tant qu'État équivaut à déclarer qu'elle remplit les conditions du statut d'État prévues par le droit international. Si ces conditions sont remplies, les États existants ont une obligation de reconnaissance » [notre traduction]. 
l'ordre juridique international, ${ }^{147}$ traduit une certaine consécration du droit international et, au-delà, d'un des principes structurants de ce droit : l'effectivité. ${ }^{148}$ En effet, la particularité de cette norme tient au fait qu'elle énonce des critères qui sont autant des " conditions légales [que] des conditions de fait ", ${ }^{149}$ ce qui explique aussi pourquoi l'effectivité constitue un critère opératoire unique pour certains juges afin d'établir la qualité étatique d'une entité. ${ }^{150}$

147 Ce choix est, par ailleurs, souvent assumé de manière expresse par le juge. Voir Parent et autres c. Singapore Airlines Ltd., supra note 73, para 53 : « [...] [C]'est [...] dans le droit international public qu'il y a lieu de rechercher la définition [d'État] à appliquer »; Italie c. Djukanovic, supra note 71, para 11 : «La norme internationale [prescrivant les conditions d'existence de la qualité étatique] est une norme de source coutumière, et donc, en tant que norme de droit international général, fait automatiquement partie de l'ordre juridique italien [...] » [notre traduction] ; Wang et consorts c. Office des juges d'instruction fédéraux, supra note 80, para 5.2 : «L'État se définit en droit international selon trois critères : un territoire ; une population ; un gouvernement effectif et indépendant [...] »; République démocratique du Timor Oriental, Fretilin et autres. c. Pays-Bas, supra note 71, 74, para 3 : «La décision [quant à la qualité d'État du Timor oriental] doit être prise sur le fondement des critères factuels [...] posés par le droit international » [notre traduction].

148 La pratique de la reconnaissance procédurale a ainsi pu être qualifiée de « de factoïsme ». Voir à ce titre Brownlie, supra note 9, 204. Plus généralement, au-delà de l'adage ex factis jus oritur servant à décrire certains concepts et institutions de droit international, nombreux sont les auteurs ayant insisté sur la « force de l'effectivité » en droit international. Voir R. Kolb, Théorie du droit international, 2 è éd. (2013), 465-467.

149 Ruiz Fabri, Genèse et disparition de l'État à l'époque contemporaine, supra note 46, 164.

150 Certaines jurisprudences font ainsi reposer le test de la qualité étatique d'une entité sur le seul critère de l'effectivité ou du contrôle gouvernemental effectif. Voir à ce titre Ungar et autres c. Organisation de libération de la Palestine, supra note 74 , para 5 : «En pratique, le troisième élément [relatif à l'existence d'un gouvernement effectif] est la condition la plus importante de la qualité étatique. [...] Les premier, deuxième et quatrième éléments dépendent [du troisième] ou [...] [sont] subsumés sous [celui-ci] » [notre traduction]. C'est notamment le cas des juridictions françaises. Voir Clerget c. Représentation commerciale de la République démocratique du Viêt-Nam, supra note 71, $1123:$ : [...] [L']effectivité de la République démocratique du Viêt-Nam, ainsi que des pouvoirs de son gouvernement, ne peut pas être déniée [...] »; Clerget c. Banque commerciale pour l'Europe du Nord et Banque du commerce extérieur du Vietnam, supra note 79, $524:$ : [...] [T] ous ces faits sont suffisamment révélateurs de l'effectivité de la [République démocratique du Viêt-Nam] [...] »; Strategic Technologies c. Procurement Bureau of the Republic of China - Ministry of National Defence, 
À ce stade du raisonnement et au vu de ce qui précède, il convient de se demander si la reconnaissance procédurale n'a de reconnaissance que le nom. Il semblerait que contrairement à la reconnaissance de jure, elle ne constitue pas une "norme-transformateur ${ }^{151}$ La reconnaissance de jure autorise, en effet, la naissance d'un sujet de droit dans l'ordre juridique interne. ${ }^{152}$ S'il fallait encore le rappeler, cette reconnaissance transforme le fait - mieux dit, la proposition de fait - et l'insère dans le droit. Au contraire, la reconnaissance procédurale ne semble qu'attester le fait dans sa réalité : elle étudie, examine, connaît simplement le fait. Or, comme certains l'ont soutenu : «il importerait [...] de distinguer les simples constatations cognitives des reconnaissances » ${ }^{153}$ ou encore, "l'acte de reconnaissance est perçu comme quelque chose de plus qu'une simple connaissance $» .{ }^{154}$ Ontologiquement, les deux reconnaissances sembleraient donc pour le moins dissemblables. Une approche positiviste, si elle ne constitue pas nécessairement la panacée, peut permettre d'aborder la question de manière plus globale à défaut d'y répondre parfaitement. La vision kelsénienne de l'ordre juridique dans son ensemble permet, en effet, de relativiser la distinction d'ordre essentiel qui existerait entre les deux mécanismes. Il n'y aurait ainsi « dans le domaine du droit [...] aucun fait absolu ou [...] évident, aucun fait en soi, mais uniquement des faits établis par l'autorité compétente au cours d'une procédure prescrite par l'ordre juridique ». ${ }^{155}$ Cette conception peut sans difficulté être appliquée à nos deux engeances de reconnaissance. Dans le cadre de la reconnaissance procédurale, le juge (autorité habilitée par une norme) procède à une application du droit (procédure prescrite par une norme) pour établir des faits juridiques. C'est ainsi au niveau de cette qualification que le juge ferait passer le fait dans le monde du droit. ${ }^{156} \mathrm{Si}$ l'on adhère à cette perspective, le prisme de la reconnaissance $d e$ jure est également présent dans le cadre de la reconnaissance procédurale. Il s'ensuivrait que les deux mécanismes pourraient entrer dans ce modèle

supra note 76, $4:$ : [...] [L]a République de Chine $[\ldots]$ constitue en fait un État souverain et indépendant $[\ldots] »$.

151 Kolb, Théorie du droit international, supra note 148, 465.

152 Cassese, International Law, supra note 44, 72.

153 Münch, supra note 12, 163.

154 Blix, Contemporary aspects of recognition, supra note 43, 637 [notre traduction].

155 Kelsen, Recognition, supra note 11, 606.

156 Santulli, supra note 48, 127 : «[...] [L]orsque les trois éléments constitutifs de l'État sont réalisés, l'État naît. Les règles qui définissent le statut de l'État envisagent l'État-factuel, mais leur effet est la constitution d'une situation juridique ». 
conceptuel de la notion d'institution juridique, dans la mesure où ils instituent un fait réel en un fait juridique. Cette conclusion putative sur la nature juridique de la reconnaissance procédurale autorise peut-être à pousser davantage la réflexion et à en envisager les effets juridiques. ${ }^{157}$

\section{Effets et fonctions de la reconnaissance procédurale d'État}

L'étude de la nature et des modalités d'application d'un mécanisme juridique doit nécessairement être suivie d'une analyse de la situation juridique qui découle de sa mise en ouvre puisqu'elle en fondera les effets de droit. Ainsi, l'octroi de la qualité étatique par le biais de la reconnaissance procédurale $\mathrm{a}$, en principe, pour conséquence juridique première de conférer le statut étatique à l'entité visée. Il apparaît, à cet égard, que l'État recon$n u$ procéduralement peut potentiellement bénéficier de toute une gamme de prérogatives (A), lesquelles trouvent leur pendant dans l'existence de certaines obligations $(\mathrm{B})$.

\section{A. Le tempérament à la portée limitée des effets juridiques}

Il a été dit que la reconnaissance de jure était la référence et qu'il était « aisé d'apprécier les conséquences juridiques de cette reconnaissance qui demeure la plus complète [:] [e]lle est un [acte] selon lequel l'ensemble des rapports juridiques internationaux qu'entretiennent d'ordinaire les États [...] s'applique [également] entre l'entité qui reconnaît et l'entité reconnue ». ${ }^{158}$ Partant, la question qui peut se poser est celle des effets juridiques procédant de l'opération étudiée au regard de ceux découlant de la reconnaissance de jure. À cet égard, si la reconnaissance procédurale telle qu'accor-

157 Sur l'impossibilité relative de subsumer un mécanisme juridique sous une catégorie juridique figée et sur l'utilité d'une analyse de celui-ci sous l'angle de ses effets juridiques, voir B. Simma et R. Abraham, Opinion dissidente commune, in Souveraineté sur Pedra Branca/Pulau Batu Puteh, Middle Rocks et South Ledge (Malaisie/Singapour), arrêt, CIJ Rec. 2008, 12, 121, para. 16 : « À vrai dire, il n'est pas de première importance $[\ldots]$ [d'avoir] recours [...] à telle ou telle catégorie ou qualification juridique, lesdites catégories n'étant souvent pas, il faut le reconnaître, séparées les unes des autres de façon étanche. [...] [C]e qui importe surtout est de savoir quels effets le droit [...] attache à tel ou tel [mécanisme] [...] plutôt que de choisir entre telle ou telle expression apte à qualifier le processus juridique qui conduit de la cause à la conséquence ».

158 Jennings, supra note 2, 354, [notre traduction]. 
dée par le juge a naturellement des effets limités au litige présenté devant lui (2), il apparaît que la palette des effets de droit procédant de celle-ci est extrêmement large (1).

\section{L'étendue des droits accordés}

L'immunité de juridiction est l'une des conséquences découlant ordinairement de la reconnaissance de jure de l'État étranger. ${ }^{159}$ Celle-ci suppose, en termes prosaïques, que l'État étranger, de par sa souveraineté, est en droit ${ }^{160}$ de ne pas être attrait devant les tribunaux étrangers. Or, il apparaît que les affaires témoignant de la mise en ouvre du mécanisme de reconnaissance procédurale et ayant trait à la question de l'immunité de juridiction sont légion. Une affaire ayant fait grand bruit aux États-Unis peut être citée pour exemple : Ungar et autres c. Organisation de libération de la Palestine. ${ }^{161}$ En l'espèce, le demandeur souhaite engager la responsabilité de l'Autorité palestinienne pour la mort d'un Américain causée par une attaque terroriste en territoire israélien. En appel, l'Autorité palestinienne, se revendiquant agent de l'État de Palestine, avance comme moyen de défense - tout comme en première instance - son droit à l'immunité de juridiction. En définitive, la Cour conclut à l'absence de qualité étatique de la Palestine et dénie, à ce titre, au défendeur la possibilité de se prévaloir de l'immunité de juridiction. Cette jurisprudence démontre, a contrario, qu'une entité non reconnue de jure mais reconnue procéduralement comme État, bénéficie de l'immunité de juridiction comme dans l'affaire canadienne préalablement étudiée, Parent et autres $c$. Singapore Airlines. ${ }^{162} \mathrm{La}$ possibilité d'étendre cette prérogative - traditionnellement accordée aux États reconnus de jure - aux entités non reconnues mais constituant en réalité des États souverains est, par ailleurs, pratiquée de longue date. ${ }^{163}$ De la

159 Kolb, Théorie du droit international, supra note 148, 680.

160 Sur la fonction de l'immunité de juridiction, voir C. Kessedjian, Droit du commerce international (2013), 168, para 313 : «L'immunité permet d'empêcher l'interférence d'un État, par le truchement de ses tribunaux, avec les activités d'un autre État et de ses représentants (par in paret non habet imperium) ».

161 Supra note 74.

162 Supra note 73.

163 Tribunal mixte de première instance d'Alexandrie, C. Tavoularidis \& $\mathrm{C}^{\circ}$ et The Equitable Trust of New York c. The National Navigation of Egypt, 8 novembre 1927 [Égypte], 17 Revue de droit maritime comparé (1928) 340, 340-341 : « [...] [L]e droit des gens admet que de l'indépendance et de la souveraineté des États il résulte qu'un Êtat ne peut être justiciable des tribunaux d'un autre État [...]. [...] 
même manière, l'immunité d'exécution a pu être accordée par le juge à un État reconnu procéduralement. ${ }^{164}$

Il est des prérogatives accordées aux États reconnus de jure qui, sans procéder directement de règles coutumières internationales, sont prévues par le droit interne de chaque État. Ces règles sont, en général, exorbitantes du droit commun et seront, de la même manière, applicables aux États reconnus procéduralement. La jurisprudence française est pourvoyeuse, dans ce cadre, d'une décision relativement récente et tout à fait audacieuse, puisqu'elle est parvenue à embarrasser le Quai d'Orsay : Strategic Technologies c. Procurement Bureau of the Republic of China. ${ }^{165}$ Strategic Technologies, société de droit singapourien, obtient un jugement favorable de la Cour suprême de Singapour, le 10 décembre 2002, qui condamne le bureau d'approvisionnement du ministère taïwanais de la défense pour non-respect de ses obligations contractuelles envers la première. Le 27 juillet 2009, l'entreprise singapourienne demande l'exécution du jugement en France devant le Tribunal de grande instance de Paris et l'assignation est directement transmise au ministère taïwanais de la défense. En date du 9 septembre 2010, le juge de l'exécution rend une ordonnance jugeant que l'assignation en demande d'exequatur est nulle car elle n'a pas été signifiée dans les formes requises. Lordonnance est confirmée en appel le 30 mars 2011. En effet, pour les deux juges, il y a vice de procédure en ce que l'acte d'assignation à un État devait nécessairement être délivré par la voie diplomatique. ${ }^{166}$ Or, l'assignation a été directement transmise au défendeur. Ainsi, pour justifier de l'applicabilité de cette disposition, la Cour d'appel a dû reconnaître procéduralement Taïwan comme État étranger, ce qu'elle fait en

La circonstance que le gouvernement en cause, en l'espèce le gouvernement soviétique, n'est pas reconnu par le gouvernement égyptien, n'autorise pas les juges à méconnaître à cet État les prérogatives qu'il exerce en fait comme État souverain $[\ldots] »$.

164 Clerget c. Représentation commerciale de la République démocratique du ViêtNam, supra note 71 ; Clerget c. Banque commerciale pour l'Europe du Nord et Banque du commerce extérieur du Vietnam, supra note 79.

165 Supra note 76.

166 Code de procédure civile [France], Art. 684, al. 2 : «L'acte destiné à être notifié à un État étranger, à un agent diplomatique étranger en France ou à tout autre bénéficiaire de l'immunité de juridiction est remis au parquet et transmis par l'intermédiaire du ministre de la justice aux fins de signification par voie diplomatique ». 
déclarant que "la République de Chine non reconnue par la France, constitue en fait un État souverain et indépendant $[\ldots] »{ }^{167}$

Les exemples peuvent être déclinés à l'infini. ${ }^{168}$ Ils nous permettent d'affirmer que l'État reconnu procéduralement bénéficie potentiellement de tout droit dérivant du statut d'État en droit interne.

\section{Des effets limités au cas de l'espèce}

Si les effets juridiques pouvant être inférés de la reconnaissance procédurale et de la reconnaissance de jure sont, dans leur essence, dans leur nature, identiques, il existe toutefois une différence qui relève des modalités de l'acte de reconnaissance et qui induit une différence au niveau des conséquences juridiques. La reconnaissance de jure est de la compétence exclusive de l'exécutif d'un État, tandis que la reconnaissance procédurale est prononcée par le juge dans la sphère délimitée d'une instance. Il s'ensuit que si la reconnaissance de jure a un effet intersubjectif entre l'État reconnaissant et l'État reconnu avec le déploiement de toutes les conséquences juridiques propres au statut d'État, la reconnaissance procédurale n'a logiquement qu'un effet inter partes. Partant, elle n'engage en principe pas l'exécutif ${ }^{169}$ et ses conséquences juridiques sont limitées à un seul lien juridique. Effectivement, les affaires étudiées plus haut n'intéressent souvent qu'un seul rapport de droit, et n'est donc souvent accordé qu'un seul effet juridique : soit l'immunité de juridiction, soit le droit à l'entraide judiciaire, soit un droit exorbitant en matière d'assignation, etc. ${ }^{170}$

167 Strategic Technologies c. Procurement Bureau of the Republic of China - Ministry of National Defence, supra note 76, 4-5.

168 Ce constat peut être systématisé en fonction de la nature des effets accordés. Évidemment, dans la mesure où le cadre de la reconnaissance procédurale est celui de l'instance, la plupart des effets sont procéduraux ou mixtes (p. ex., respectivement, jus standi et immunités), mais ils peuvent également être substantiels (octroi d'effets aux actes étrangers dans l'État du for).

169 Charpentier, Reconnaissance, supra note 22, para 23.

170 En tout état de cause, l'octroi ou le refus de la qualité étatique par le biais d'une reconnaissance procédurale a toutefois, en pratique, un influence normative dépassant le cadre de l'espèce en tant que précédent potentiel - ayant à tout le moins une autorité persuasive - pour les décisions juridictionnelles ultérieures. En témoigne la décision Gilmore et autres c. Autorité palestinienne intérimaire autonome et autres (supra note 72) qui renvoie à la décision de non-reconnaissance procédurale rendue dans l'affaire Ungar et autres c. Organisation de libération de la Palestine (supra note 74). 


\section{B. Le rapport entre obligations étatiques et fonction de la reconnaissance procédurale}

Si la reconnaissance procédurale a pour effet d'octroyer des droits à l'entité dépourvue de reconnaissance de jure, il est logique qu'elle induise corrélativement l'imposition d'obligations dans le chef de l'entité en question. En effet, selon l'adage jus et obligatio correlata sunt, à chaque droit correspond une obligation et vice versa (1). Les effets souhaités de l'institution de la reconnaissance procédurale permettront, en définitive, d'en éclairer la fonction laquelle tiendrait à une finalité générale de bonne administration de la justice (2).

\section{Les obligations de l'État reconnu procéduralement}

Cette éventualité pourrait, en théorie, trouver application dans bon nombre de situations, bien qu'il s'avère difficile de mettre au jour des affaires spécifiques où l'obligation de l'État reconnu procéduralement aurait été soulevée. ${ }^{171} \mathrm{Si}$ les décisions de juridictions nationales n'offrent que très peu d'apports quant aux devoirs imposés à l'État (ou au gouvernement) reconnu procéduralement, il est possible de déceler dans la jurisprudence arbitrale des solutions qui pourraient être transposées aux situations portées devant le juge interne. En effet, dans plusieurs sentences, l'arbitre, par le truchement d'un raisonnement juridique identique à celui éprouvé dans le cadre des décisions internes, a reconnu procéduralement des gouvernements non reconnus de jure et a appelé au respect de leurs obligations en tant que tels $;{ }^{172}$ la violation de celles-ci devant, en principe, engager leur responsabilité.

La doctrine a, par ailleurs, eu l'occasion de s'exprimer sur la question en étudiant la pratique étatique en la matière. La retranscription de la déclaration d'un ancien ministre britannique des affaires étrangères lors des mouvements indépendantistes qui secouèrent l'Amérique espagnole au début du dix-neuvième siècle a pour avantage d'attester de l'idée que la responsabilité des États non reconnus de jure était admise de longue date:

171 Cette situation découle largement du fait que l'État reconnu procéduralement brandira son droit à l'immunité afin de ne pas voir sa responsabilité engagée.

172 Notamment lorsque leurs actes ont des effets sur la situation juridique d'individus étrangers. Voir Affaire Tinoco (Royaume-Uni c. Costa Rica), supra note 111 ; Affaire George W. Hopkins (États-Unis d'Amérique c. États-Unis du Mexique), 31 mars 1926, 4 RSA 41. 
Or, ou la mère patrie des colonies espagnoles est restée responsable d'actes sur lesquels elle ne pouvait plus exercer l'ombre même d'une autorité, ou les habitants de ces pays, dont l'existence politique indépendante était, en fait, établie mais auxquels l'acceptation de cette indépendance avait été refusée, [...] devaient être placés dans une situation telle qu'ils n'encourussent de responsabilité pour aucun de leurs actes, ou bien devaient être frappés, pour les actes qui pourraient donner lieu à des plaintes de la part d'autres nations, des pénalités réservées aux pirates ou aux individus hors la loi. Si la première de ces alternatives - l'irresponsabilité totale d'États non reconnus - est trop absurde pour être soutenue, et si la deuxième alternative - traiter leurs habitants en pirates et en hommes hors la loi - est trop monstrueuse pour être appliquée $[\ldots]$, il ne restait $[\ldots]$ qu'à reconnaître en temps utile leur existence politique comme États et à les introduire ainsi dans le cercle des droits et devoirs que les nations civilisées sont tenues de respecter et ont le droit réciproquement d'exiger les unes des autres dans leurs rapports mutuels. ${ }^{173}$

\section{Le souci de bonne administration de la justice}

L'absence de différences notoires entre les droits et obligations dont jouit l'État reconnu de jure et ceux de l'État reconnu procéduralement autorise à s'interroger sur la raison d'être du mécanisme de la reconnaissance par le juge. Les développements précédents peuvent apporter un début de réponse.

Il apparaît que le mécanisme de reconnaissance procédurale est - nous l'avons déjà évoqué - mis en mouvement lorsqu'il y a refus ou absence de toute reconnaissance de jure. Dans ce contexte, la reconnaissance procédurale a une finalité première : obvier à un résultat inadmissible, déraisonnable, voire absurde ${ }^{174}$ ou plus rarement combler un vacuum juris. ${ }^{175} \mathrm{La}$ doctrine,

173 G. Canning, Note of Mr. Secretary to the Chevalier de Los Rios relative to Spanish America, 12 British and Foreign State Papers (1825), 909, 912-913 [traduction, Williams, supra note 31,225$]$.

174 Verhoeven, Les relations internationales, supra note 2, 66 : « [La reconnaissance procédurale est conçue comme un] [...] expédien[t] [...] pour prévenir des conséquences qui frisent l'absurdité ».

175 Loin de nous prononcer en faveur de la possibilité théorique de l'existence d'un non-droit, rien n'empêche un juge de prononcer un non liquet dans cette situation. 
encore une fois, a su faire écho à ces écueils. ${ }^{176}$ Ces deux difficultés peuvent être exemplifiées très simplement. Posons un litige dans lequel est engagée la responsabilité des autorités contrôlant effectivement Taïwan, largement non reconnu de jure. Il y aurait incontestablement un certain vide juridique - pouvant prendre la forme d'un déni de justice ${ }^{177}$ - si un tribunal devait décider de l'impossibilité de soulever la responsabilité du défendeur non reconnu de jure en tant qu'État car celui-ci n'aurait pas de personnalité juridique. Et, un résultat déraisonnable serait obtenu si le juge devait statuer que la responsabilité de la République populaire de Chine était engagée alors même que, dans les faits, celle-ci n'exerce aucune autorité effective sur le territoire en question. ${ }^{178}$ Entre ces deux extrêmes, la reconnaissance procédurale est donc la reconnaissance du pragmatisme juridique. ${ }^{179}$

La reconnaissance procédurale aurait, par conséquent, l'avantage de tenir compte de certaines considérations ayant trait à la bonne administration de la justice. ${ }^{180}$ Ces considérations sont particulièrement prégnantes lorsque la validité de certains actes juridiques de l'État non reconnu de jure se pose. ${ }^{181}$ A. Aust déclare ainsi, à l'appui de nombreuses affaires, que les tribunaux nationaux donnent effet aux actes et décisions d'autorités publiques d'un État non reconnu de jure lorsqu'ils intéressent des aspects es-

176 P. L. Hsieh, An Unrecognized State in Foreign and International Courts: the Case of the Republic of China on Taiwan, supra note 128, $814:$ : [...] [L]es obstacles politiques ne devraient pas entraver la finalité fondamentale des tribunaux, laquelle est de rendre justice » [notre traduction].

177 Nous devons relever que ce déni de justice pourrait toutefois être caractérisé si le défendeur devait brandir son droit à l'immunité découlant de sa reconnaissance procédurale.

178 C'est le résultat déraisonnable atteint par une juridiction britannique dans le cas de la République démocratique allemande non reconnue de jure par l'exécutif britannique. Pour contourner la difficulté, il est jugé que l'URSS, reconnue par la Couronne britannique, exerce un contrôle effectif - ce qui est démenti par les faits - sur la RDA, et donc que les actes émanant de l'Allemagne de l'Est relève de la responsabilité de l'Union soviétique : Chambre des Lords, Carl-Zeiss Stiftung c/ Rainer \& Keeler Limited, 15 mai 1966, 43 ILR 23 [Royaume-Uni].

179 Cour d'appel de New York, Boris N. Sokoloff c. National City Bank of New York, 25 novembre 1924, 2 ILR 44, 47 : «Juridiquement, un gouvernement non reconnu peut être considéré comme n'étant pas un gouvernement du tout [...]. En pratique, néanmoins, dans la mesure où les notions juridiques sont rarement, voire jamais, poussées jusqu'au bout de leur logique, [...] [elles sont] sujette[s] aux limitations - qui s'imposent d'elles-mêmes - de bon sens et de justice » [notre traduction].

180 Degan, Création et disparation de l'État à la lumière du démembrement de trois fédérations multiethniques en Europe, supra note 50, 248.

181 Couveinhes-Matsumoto, supra note 17, 274-275, para 266. 
sentiels de la vie quotidienne, comme les naissances, les mariages, les divorces et les décès $;^{182}$ mais également des relations économiques et commerciales. Cette vision est embrassée par une grande partie de la doctrine, ${ }^{183}$ notamment parce qu'elle est dans l'intérêt respectif des parties. ${ }^{184}$ Très pragmatique, cette solution n'est pas toute récente, car elle a pu être consacrée dans la jurisprudence américaine dès le dix-neuvième siècle. Dans la décision Texas c. White et autres, ${ }^{185}$ la Cour suprême américaine a ainsi déclaré ce qui suit :

[I]l peut être dit, [...] avec suffisamment de justesse, que les actes nécessaires à la paix et au bon ordre parmi les citoyens, comme par exemple les actes sanctionnant et protégeant l'institution du mariage et les relations domestiques, ceux ressortissant à la filiation, ceux régissant la cession et le transfert de biens meubles et immeubles, ceux permettant d'apporter réparation pour des préjudices corporels ou matériels, et les autres actes similaires, qui seraient valides s'ils émanaient d'un gouvernement légitime, doivent être considérés en général comme valides lorsqu'ils émanent d'un gouvernement qui existe en fait, bien qu'il soit illégitime [...]. ${ }^{186}$

\section{Conclusion}

Expression forgée a priori à des fins d'investigation, la reconnaissance procédurale ne renvoie pas moins à un phénomène juridique tout à fait tangible. À ce titre, il ne fait aucun doute - nous le réitérons - qu'elle ne répond pas à la définition de la notion de reconnaissance telle qu'elle est conçue en

182 A. Aust, Handbook of International Law, 2 è éd. (2011), 21.

183 Bierzaneck, supra note 5, 125 : «Sous la pression des besoins de la vie, les tribunaux de différents pays ont trouvé une solution à la majorité des questions pratiques soulevées par la non-reconnaissance »; R. Ranjeva et Ch. Cadoux, Droit international public (1992), 94 : " Pour des considérations de bonne administration de la justice, les actes de l'État non reconnu peuvent être pris en compte par les juridictions des États tiers et faire objet d'application dès lors que les litiges n'ont qu'un caractère privé et ne mettent pas [...] en cause des problèmes de relations d'État à État ».

184 Daillier et al., supra note 35, 623, para 366 : «[...] [C]ette attitude est conforme aux exigences de bonne administration de la justice et aux intérêts des parties. Ce souci de réalisme se retrouve dans les jurisprudences internes [...]».

185 Cour suprême, Texas c/ White, 12 avril 1869, 74 United States Reports 700 [ÉtatsUnis].

186 Ibid., para 128 [notre traduction]. 
théorie et qu'elle opère en pratique dans l'ordre juridique international. ${ }^{187}$ Il apparaît toutefois que dans l'ordre juridique interne, au regard de sa nature, de ses effets et de sa fonction, la reconnaissance procédurale semble se rapprocher à bien des égards de la reconnaissance de jure en octroyant la qualité étatique à une entité qui la revendique et le statut qui en découle. Et, en définitive, en faisant nôtre la conception selon laquelle il n'y a de droits et obligations que ceux qui sont sanctionnés par le juge, ${ }^{188}$ alors la différence entre l'État reconnu procéduralement et l'État reconnu de jure se fait plus ténue...

187 Verhoeven, Les relations internationales, supra note 2, 23 : « [La reconnaissance procédurale] est étrangère [...] aux formes de la reconnaissance. Elle vise en effet l'autonomie dont dispose le juge dans le règlement de conflits qui mettent en cause, au moins en apparence, des actes de droit international public, et non la forme sous laquelle est exprimée la reconnaissance. [...] [La reconnaissance] n'est [...] valable en droit des gens que si elle émane d'un organe habilité à représenter l'État dans les relations internationales. Le juge ne disposant [...] pas d'une telle qualité, sa reconnaissance ne saurait être constitutive d'une reconnaissance [même] implicite de l'État dans les rapports internationaux » [nous soulignons].

188 O. W. Holmes Jr., La voie du droit (2014), 3-4 : «Répétons-le : les droits et obligations [...] dont s'occupe la doctrine ne sont rien d'autre que des prophéties. [...] $[\mathrm{L}] \mathrm{a}$ théorie se retrouve à placer la charrue avant les bœufs, et [à] imaginer que les droits et obligations existeraient de manière indépendante des conséquences de leur violation $[\ldots]$. Pourtant, $[\ldots]$ une obligation juridique n'est $[\ldots]$ rien d'autre que la prédiction de ce que, si un [sujet] commet ou omet certaines choses, il devra subir [...] le jugement d'un tribunal - et il en va de même des droits ». 